\title{
Damage Detection for Continuous Bridge Based on Static-Dynamic Condensation and Extended Kalman Filtering
}

\author{
Haoxiang He, Yongwei Lv, and Enzhen Han \\ Beijing Laboratory of Earthquake Engineering and Structural Retrofit, Beijing University of Technology, Beijing 100124, China \\ Correspondence should be addressed to Haoxiang He; hhx7856@163.com
}

Received 2 January 2014; Revised 3 April 2014; Accepted 7 April 2014; Published 30 April 2014

Academic Editor: Ying Lei

Copyright ( 2014 Haoxiang He et al. This is an open access article distributed under the Creative Commons Attribution License, which permits unrestricted use, distribution, and reproduction in any medium, provided the original work is properly cited.

\begin{abstract}
As an effective and classical method about physical parameter identification, extended Kalman filtering (EKF) algorithm is widely used in structural damage identification, but the equations and solutions for the structure with bending deformation are not established based on EKF. The degrees of freedom about rotation can be eliminated by the static condensation method, and the dynamic condensation method considering Rayleigh damping is proposed in order to establish the equivalent and simplified modal based on complex finite element model such as continuous girder bridge. According to the requirement of bridge inspection and health monitoring, the online and convenient damage detection method based on EKF is presented. The impact excitation can be generated only on one location by one hammer actuator, and the signal in free vibration is analyzed. The deficiency that the complex excitation information is needed based on the traditional method is overcome. As a numerical example, a three-span continuous girder bridge is simulated, and the corresponding stiffness, the damage location and degree, and the damping parameter are identified accurately. It is verified that the method is suitable for the dynamic signal with high noise-signal ratio; the convergence speed is fast and this method is feasible for application.
\end{abstract}

\section{Introduction}

In civil engineering, the structures such as buildings and bridges will inevitably be damaged due to varied natural hazards and environmental effect. The damage includes the change of the materials property and the geometrical feature in the total structure or local members, besides the deterioration on the stiffness, the strength, the boundary, and the connection conditions. Therefore, intelligent heath monitoring and damage detection for structures become an important technology to study, and detecting and predicting the structural damage in time are necessary for future engineering. The traditional structural damage detection mainly refers to identifying the change of physical parameters such as stiffness, frequency, and damping or recognizing the damage patterns through analyzing the characteristics of the input and output signals in time domain or frequency domain; the finite element model or the dynamic equations are often needed and the damage characteristics and degree are determined finally [1].
In general, the principal stages and targets of damage detection or structural health monitoring include four levels: (1) the determination of the presence of damage in the structure; (2) the determination of the damage location in the structure; (3) the quantification of the severity of the damage; (4) the prognosis of the remaining service life of the damaged structure. The damage detection methods mainly include four types: (1) methods based on modal parameters, such as the change of the frequencies, modal shapes, and transfer function, modal strain energy method (MSE), and flexibility matrix method (FM) [2]; (2) methods based on signal processing, such as wavelet analysis, wavelet packet analysis, and empirical mode decomposition (EMD) [3]; (3) methods based on intelligent computing and signal analysis, such as artificial neural network method, statistical pattern recognition, fuzzy theory, and genetic algorithm [2]; (4) methods on physical parameter identification, such as Kalman filter method and the generalized Tikhonov regularization method [4]. In recent 20 years, various damage detection methods have developed promptly, but each method has its own 
limitation. The modal parameter is insensitive to minor damage and a great many sensors are needed to finish modal analysis. As the modal method, the limited amount of sensors in practice also impede the application of MSE or FM. It needs numerous hypothetical damage patterns for signal processing and intelligent computing, and these patterns are always simulated in complex finite element analysis. For the methods above, the damage degree is determined according to the comparison for different cases or relative parameters but not obtained directly based on dynamic equations or physical parameters. In contrast, the methods on physical parameter identification aim to recognize the physical parameters in the system mechanism and the results are adjusted constantly according to the inputs and outputs.

The physical identification, viewed as an inverse problem, aims to determine a set of physical parameters of the structure. The physical parameters might be considered as the stiffness, damping, and mass of the structure. These estimated parameters can then be used, among other quantities, to predict the damage information or to assess the structural conditions. In essence, physical identification can be considered as an optimization process in which the objective is to identify a model of a system so that its predicted response to a given input is close enough to the measured response from the real system. The major method of physical identification aims to estimate the system's physical parameters using directly the time histories of the observed structural response and tries to create a mechanical model that simulates such response as closely as possible. Among physical identification method, the important method includes the Kalman filters, leastsquares methods, the particle filter, and sequential Monte Carlo method.

The extended Kalman filtering (EKF) algorithm is a very effective method to identify system parameter. As a type of recursive algorithm, Kalman filtering realizes the linear unbiased minimum variance estimation to the system according to the discrete observation data [4]. The normal EKF requires the complete signals of both the input excitation and the structural response. However, in practice, the time history of dynamic input forces, such as wind and traffic, is often difficult to be directly measured. In addition, the structural responses about the acceleration, velocity, and displacement are all needed. In order to improve the completion requirement of the inputs and outputs, various EFK-based approaches have been widely investigated. Wang and Halder developed a method combining the iterative least-squares procedure with unknown input excitations and the EKF with a weighted global iteration [5-7]. The unique feature of this method is that it does not require response measurements at all DOFs. The efficiency, accuracy, and robustness of the method were verified by numerical examples. Ghosh et al. proposed two novel forms of the EKF, which do not require the computation of Jacobian matrices at any stage [8]. Other advantages of the methods over the conventional EKF are superior numerical accuracy and considerably less sensitive to the choice of time-step sizes. Meiliang and Smyth used the unscented Kalman filter (UKF) to estimate the states and identify the parameters of a highly nonlinear system [9]. The results indicate that the UKF can achieve better state estimation and parameter identification than the EKF. Yang et al. proposed an EKF with unknown inputs to identify the structural parameters and the unmeasured excitations online, but the analytical recursive solutions are obtained by rather complex mathematical derivations [10-12]. Recursive solutions for structural parameters and unknown excitation are derived simultaneously. As an improved method, an algorithm based on sequential application of the extended Kalman estimator and least-squares estimation is proposed by Lei et al. for the identification of structural parameters and unknown excitations [13-16].

However, among the examples in the above research, the damage detection based on EKF is mostly used in shear structural model only considering the shear effect or a simple bending structure such as simply supported beam; there are few studies on the parameter identification of complex structure mainly with bending shear deformation [17-22]. The main reasons are as follows. (1) The traditional algorithms need to obtain all the rotational responses of the bending shear structure, so the DOFs are large and the state equation is complex and even hard to be established. Meanwhile, the rotational response is hard to be accurately measured in the actual test or monitoring. (2) In addition, in order to realize the parameter identification with part of the output information, rotational response of the bending shear structure needs to be calculated with inverse algorithm, but the calculations are complex and the precision cannot fully meet the requirements. Furthermore, many researches focus on the structural nonlinear response under strong earthquake in order to detect the accumulated damage or the hysteresis characteristics, but the slow deterioration is more important for practical inspection and monitoring because the environmental effect, fatigue, and the corrosion are the major factors for damage. Hence, the novel approach and technique aimed to use online monitoring which is needed to overcome the disadvantage of current damage detection based on EKF.

In this paper, the signals of the rotation angle in the bridge are eliminated and contained by the static-dynamic condensation method instead of using the incomplete algorithm or the inversion formula, so the dynamic state equations are simplified in the initial stage, which provide an effective technique on establishing the state equations for large and complex bridges. The detection can be carried out according to the limited amount of accelerometers. Meanwhile, for engineering requirements of bridge detection and monitoring, a damage detection method for continuous girder bridge based on EKF is proposed which merely use the free vibration generated by one hammer action or vehicle bumping. The imposition and acquisition of the vast excitations are effectively avoided, the identification accuracy is high, and the practicality and the convenience are evident.

\section{Extended Kalman Filtering Algorithm}

For the structures such as bridges and buildings, the mechanical model, that is, the state equation, according to the dynamic equation is nonlinear. Hence, the nonlinear version of the Kalman filter called extended Kalman filter (EKF) is adopted to realize linearization, and the EKF is a linear 
filter with minimum variance estimate. In the EKF, the initial state transition and observation state space models are many nonlinear functions:

$$
\begin{gathered}
\dot{X}\left(t_{i+1}\right)=f\left[X\left(t_{i}\right), t_{i}\right]+W\left(t_{i}\right), \\
Z\left(t_{i}\right)=h\left[X\left(t_{i}\right), t_{i}\right]+V\left(t_{i}\right),
\end{gathered}
$$

where $X\left(t_{i}\right)$ is the state vector at the time $t_{i}, Z\left(t_{i}\right)$ is the observation vector at the time $t_{i}$, and $W\left(t_{i}\right)$ and $V\left(t_{i}\right)$ are white Gaussian, independent random processes with zero mean and covariance matrix:

$$
Q_{i}=E\left[W\left(t_{i}\right) W\left(t_{i}\right)^{T}\right], \quad R_{i}=E\left[\begin{array}{lll}
V\left(t_{i}\right) & \left.V\left(t_{i}\right)^{T}\right]
\end{array} .\right.
$$

$X\left(t_{0}\right)$ is the system initial condition considered as a Gaussian random vector. Let $Z\left(t_{1, i}\right)=\left\{Z\left(t_{1}\right), Z\left(t_{2}\right), \ldots, Z\left(t_{i}\right)\right\}$ be a set of system measurements. The filter aim to obtain an estimate of the system's state based on these measurements. The EKF gives an approximation of the optimal estimate. The nonlinearity of the system's dynamics is approximated by a linearized version of the nonlinear system model around the last state estimate. For this approximation to be valid, this linearization should be a good approximation of the nonlinear model in the entire uncertainty domain associated with the state estimate. So the filtering equations of extended Kalman filtering are as follows.

The predicted state is

$$
\begin{aligned}
\widehat{X}\left(\frac{t_{i+1}}{t_{i}}\right)=\widehat{X}\left(\frac{t_{i}}{t_{i}}\right)+f\left[\widehat{X}\left(\frac{t}{t_{i}}\right), t\right] d t, \\
P\left(\frac{t_{i+1}}{t_{i}}\right)=\Phi\left[\widehat{X}\left(\frac{t_{i+1}}{t_{i}}\right), t_{i}, t_{i+1}\right] \\
\times P\left(\frac{t_{i}}{t_{i}}\right) \Phi\left[\widehat{X}\left(\frac{t_{i}}{t_{i}}\right), t_{i}, t_{i+1}\right]+Q\left(t_{i}\right) .
\end{aligned}
$$

The filtered equations are

$$
\begin{aligned}
G\left[\widehat{X}\left(\frac{t_{i+1}}{t_{i}}\right), t_{i+1}\right]= & P\left(\frac{t_{i+1}}{t_{i}}\right) H^{T}\left[\widehat{X}\left(\frac{t_{i+1}}{t_{i}}\right), t_{i+1}\right] \\
& \times\left\{H\left[\widehat{X}\left(\frac{t_{i+1}}{t_{i}}\right), t_{i+1}\right] \cdot P\left(\frac{t_{i+1}}{t_{i}}\right) H^{T}\right. \\
& \left.\times\left[\widehat{X}\left(\frac{t_{i+1}}{t_{i}}\right), t_{i+1}\right]+R\left(t_{i+1}\right)\right\}^{-1}, \\
\widehat{X}\left(\frac{t_{i+1}}{t_{i+1}}\right)= & \widehat{X}\left(\frac{t_{i+1}}{t_{i}}\right)+G\left[\widehat{X}\left(\frac{t_{i+1}}{t_{i}}\right), t_{i+1}\right] \\
& \times\left\{Z\left(t_{i+1}\right)-h\left[\widehat{X}\left(\frac{t_{i+1}}{t_{i}}\right), t_{i+1}\right]\right\},
\end{aligned}
$$

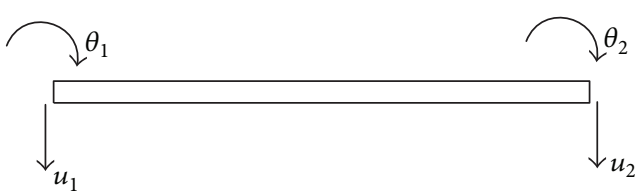

Figure 1: Beam element model.

$$
\begin{aligned}
P\left(\frac{t_{i+1}}{t_{i+1}}\right)=\{I & -G\left[\widehat{X}\left(\frac{t_{i+1}}{t_{i}}\right), t_{i+1}\right] \\
& \left.\times H\left[\widehat{X}\left(\frac{t_{i+1}}{t_{i}}\right), t_{i+1}\right]\right\} P\left(\frac{t_{i+1}}{t_{i}}\right),
\end{aligned}
$$

where $\widehat{X}\left(t_{i} / t_{i}\right)$ and $P\left(t_{i} / t_{i}\right)$ are state estimation vector and error covariance matrix at the time $t_{i}, \widehat{X}\left(t_{i+1} / t_{i}\right)$ and $P\left(t_{i+1} / t_{i}\right)$ are state estimation vector and error covariance matrix of the time $t_{i}$ to $t_{i+1}, \Phi\left[\widehat{X}\left(t_{i+1} / t_{i}\right), t_{i}, t_{i+1}\right]$ is the state-transition matrix of the time $t_{i}$ to $t_{i+1}, G\left[\widehat{X}\left(t_{i+1} / t_{i}\right), t_{i+1}\right]$ is the gain matrix, $I$ is identity matrix, and $H\left[\widehat{X}\left(t_{i+1} / t_{i}\right), t_{i+1}\right]$ is the Jacobian matrices of $h\left[X\left(t_{i+1}\right), t_{i+1}\right]$.

If the initial state vector $X\left(t_{0} / t_{0}\right)$ and initial error covariance matrix $P\left(t_{0} / t_{0}\right)$ are given and the observation vector $Z\left(t_{i}\right)$ is known, the structural physical parameters can be identified with the recursive iteration step by step of the Kalman filter equations as above. Finally, the response can also be estimated to approach the real response.

\section{Static-Dynamic Condensation Method}

As an important type of structure in civil engineering, the bridge is liable to be aging and experiencing deterioration. It is urgent to properly evaluate the structural condition and performance of these bridges under the current traffic and to determine the strategy about maintenance. The bridge structure is mainly composed of beam elements and the axial effect of the elements is generally ignored. As shown in Figure 1, a normal beam element has two nodes and each node has two degrees of freedom including rotation and a translation; thus, a beam element contains four degrees of freedom. The dynamic equation of the bridge is more complex than the shear frame because the degrees of freedom about rotation should be considered, and the rotation angle data is hard to be measured in the practical application. The condensation method is proposed to completely eliminate rotational response in this paper, and only the translational responses are required [23].

First, the deck of the bridge is divided into many elements. The mass of each element is simplified as the lumped mass on both ends and the mass on the bearing is ignored. Each mass point has one degree of freedom about translation and one degree of freedom about rotation. The mass for the translation is equal to the mass of the element; however, the mass corresponding to rotation is zero.

In order to condense stiffness more conveniently, the first and the second columns of the element stiffness matrix are written as translational stiffness and the third and the fourth columns of the element stiffness matrix are written as 
rotational stiffness. The element stiffness matrix is shown as follows:

$$
\bar{K}=\left[\begin{array}{cccc}
u_{1} & u_{2} & \theta_{1} & \theta_{2} \\
-12 k & -12 k & 6 k l & 6 k l \\
6 k l & -6 k l & 4 k l^{2} & 2 k l^{2} \\
6 k l & -6 k l & 2 k l^{2} & 4 k l^{2}
\end{array}\right]
$$

where $k=E I / l^{3}$ and $l$ is the length of the beam element.

The global stiffness matrix $\mathbf{K}$ is integrated from element stiffness matrix $\bar{K}$. K is a symmetric matrix whose first $n$ columns correspond to the translational stiffness columns of $n$ points and the later $n$ columns correspond to the rotational stiffness columns of $n$ points. The global stiffness matrix $\mathbf{K}$ is divided as follows:

$$
K=\left[\begin{array}{ll}
K_{t t} & K_{t 0} \\
K_{0 t} & K_{00}
\end{array}\right],
$$

where $K_{t t}, K_{t 0}, K_{0 t}$, and $K_{00}$ are the partitioned matrices of the matrix $\mathbf{K}$.

The damping is viewed as Rayleigh damping, and the damping matrix is as follows:

$$
C=\alpha\left[\begin{array}{cc}
M_{t t} & 0 \\
0 & 0
\end{array}\right]+\beta\left[\begin{array}{ll}
K_{t t} & K_{t 0} \\
K_{0 t} & K_{00}
\end{array}\right],
$$

where $\alpha$ and $\beta$ are the Rayleigh damping coefficients.

The mass corresponding to the rotational degree of freedom is zero, so the dynamic equation of the bridge structure is

$$
\begin{gathered}
{\left[\begin{array}{cc}
M_{t t} & 0 \\
0 & 0
\end{array}\right]\left(\begin{array}{l}
\ddot{u} \\
\ddot{\theta}
\end{array}\right)+\left\{\alpha\left[\begin{array}{cc}
M_{t t} & 0 \\
0 & 0
\end{array}\right]+\beta\left[\begin{array}{ll}
K_{t t} & K_{t 0} \\
K_{0 t} & K_{00}
\end{array}\right]\right\}\left(\begin{array}{l}
\dot{u} \\
\dot{\theta}
\end{array}\right)} \\
+\left[\begin{array}{ll}
K_{t t} & K_{t 0} \\
K_{0 t} & K_{00}
\end{array}\right]\left(\begin{array}{c}
u \\
\theta
\end{array}\right)=\left(\begin{array}{c}
P(t) \\
0
\end{array}\right),
\end{gathered}
$$

where $u$ and $\theta$ are the translational and rotational displacement, respectively, $P(t)$ is the applied load, $M_{t t}$ is diagonal matrix, and the stiffness matrix $K$ and Rayleigh damping matrix $C$ are both symmetric matrices.

In practical application, the translational response can be measured by displacement sensor, speed sensor, and acceleration sensor, but the rotational time-history response cannot be measured as there are not any corresponding sensors to the rotate displacement, rotation speed, and acceleration. However, in the damage identification with the traditional Kalman filtering algorithm, all the time-history responses of each point need to be obtained. For this problem, a method based on static and damping force condensation is proposed in this paper to eliminate rotational degree of freedom with zero mass. The specific condensed formulas are as follows.

Equation (8) is rewritten as the following two equations:

$$
\begin{gathered}
M_{t t} \ddot{u}+\left(\alpha M_{t t}+\beta K_{t t}\right) \dot{u}+\beta K_{t 0} \dot{\theta}+K_{t t} u+K_{t 0} \theta=P(t), \\
\beta K_{0 t} \dot{u}+\beta K_{00} \dot{\theta}+K_{0 t} u+K_{00} \theta=0 .
\end{gathered}
$$

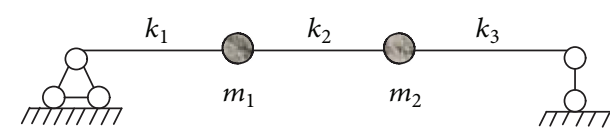

Figure 2: Simply supported beam bridge model.

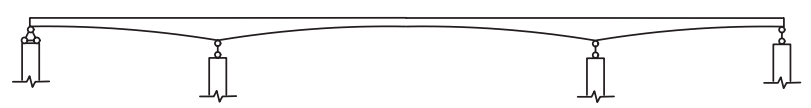

FIGURE 3: Schematic diagram of three-span continuous girder bridge.

Equation (10) is written as

$$
\beta \dot{\theta}+\theta=-K_{00}^{-1} K_{0 t}(\beta \dot{u}+u) .
$$

Substituting (11) into (9), the final dynamic equation with force and damping condensation is

$$
M_{t} \ddot{u}+C_{t} \dot{u}+K_{t} u=P(t),
$$

where $M_{t}=M_{t t}$ is the condensed mass matrix after, $C_{t}=$ $\alpha M_{t t}+\beta K_{t t}-\beta K_{t 0} K_{00}^{-1} K_{0 t}$ is condensed damping matrix, and $K_{t}=K_{t t}-K_{t 0} K_{00}^{-1} K_{0 t}$ is the condensed stiffness matrix.

Seen from (12), it is evident that the damping force and the static force can be condensed if the mass matrix, stiffness matrix, the excitations, and translation responses for each mass point are provided and the dynamic equation can be obtained. Furthermore, the dynamic modal for Kalman filtering can be established without rotational time-history response.

\section{State Equation and Observation Equation Based on Condensation Method}

The state equation and observation equation based on staticdynamic condensation method in the identification of EKF will be introduced by an example of simply supported beam. As shown in Figure 2, the length of the beam is $L$, and the beam is divided into three elements with equal length. The mass of beam is equivalent to two lumped mass points at the third of girder. Because the condensation algorithm involves matrix inversion, for simplicity, the stiffness of the three elements is assumed to be equal, $k_{1}=k_{2}=k_{3}=k=E I / l^{3}$. Then the element stiffness matrix $\frac{1}{K}$ according to formula (5) is

$$
\bar{K}=\left[\begin{array}{cccc}
12 k & -12 k & 6 k l & 6 k l \\
-12 k & 12 k & -6 k l & -6 k l \\
6 k l & -6 k l & 4 k l^{2} & 2 k l^{2} \\
6 k l & -6 k l & 2 k l^{2} & 4 k l^{2}
\end{array}\right]
$$

According to the above method on static-dynamic condensation, the global stiffness matrix $K_{t}$ only with translational degree of freedom is integrated as

$$
K_{t}=\left[\begin{array}{cc}
\frac{48 k}{5} & -\frac{42 k}{5} \\
-\frac{42 k}{5} & \frac{48 k}{5}
\end{array}\right] .
$$




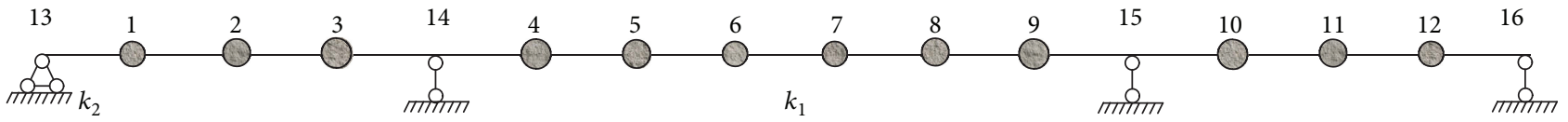

FIGURE 4: Simplified model of three-span continuous girder bridge.
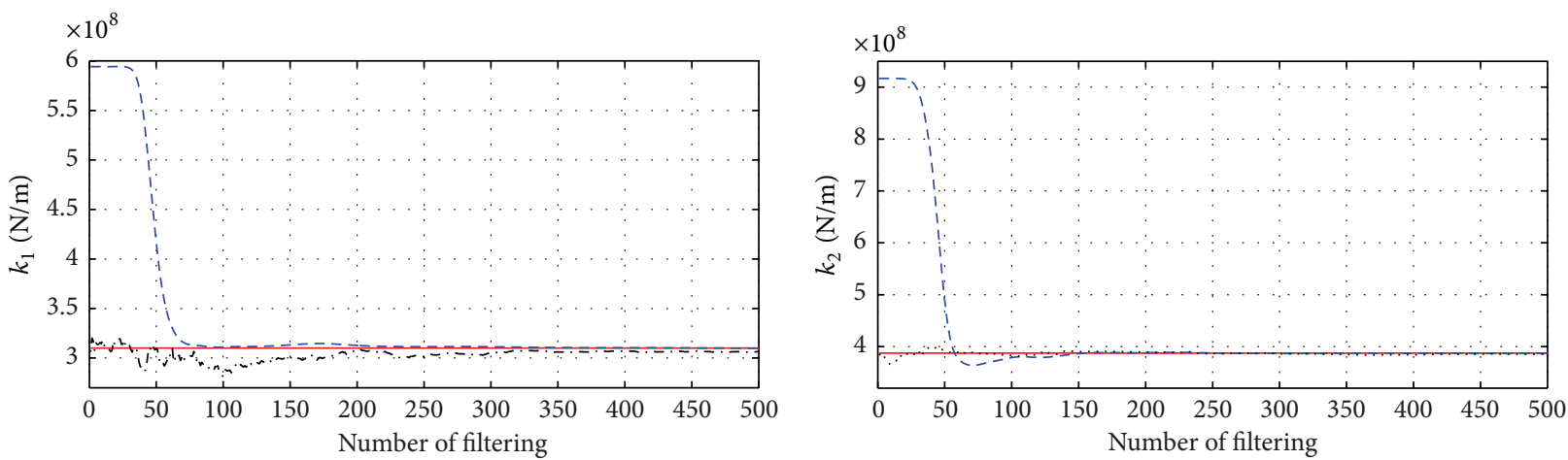

- Actual $k_{1}$ in case 1

- - - Identified $k_{1}$ with no noise in case 1

. . . - Identified $k_{1}$ with noise in case 1

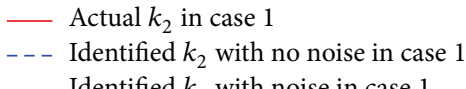

...... Identified $k_{2}$ with noise in case 1

(a)

(b)

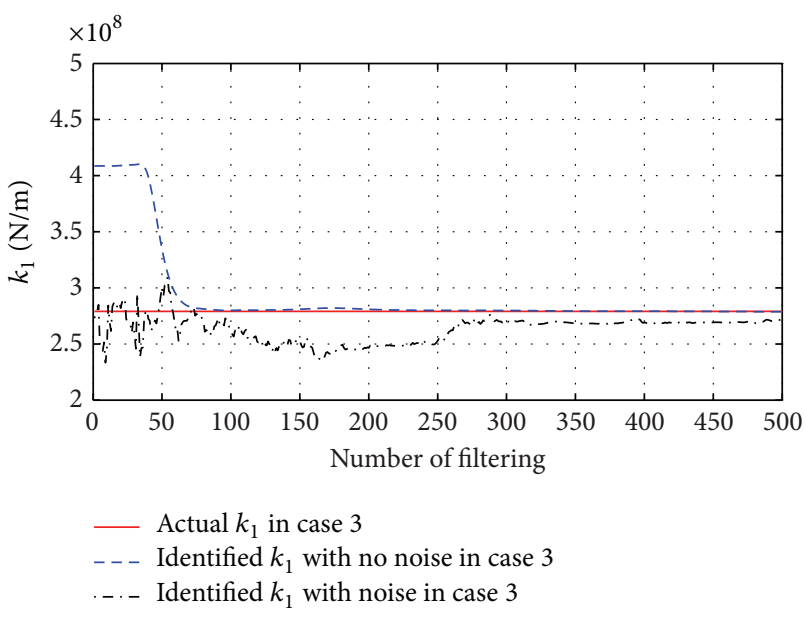

(c)

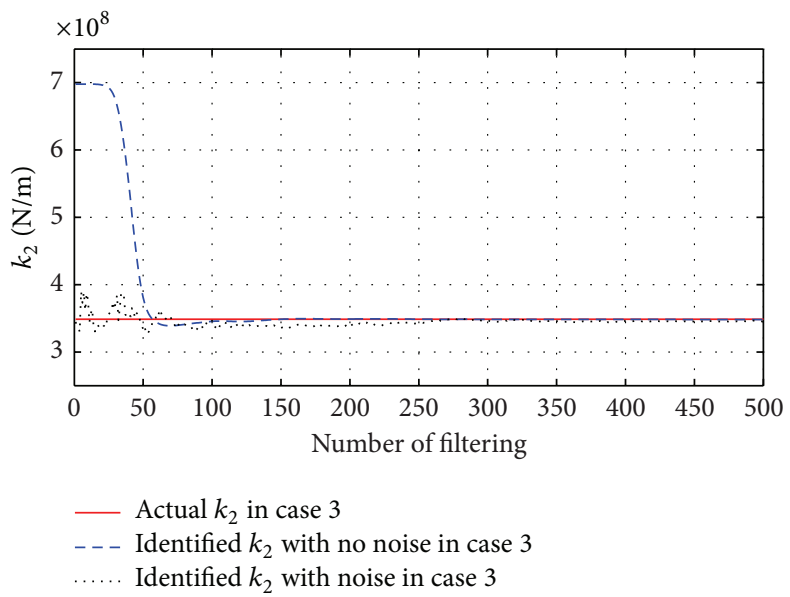

(d)

FIGURE 5: Identification results of $k_{1}$ and $k_{2}$ in case 1 and case 3 under continuous excitation.

The integrated damping matrix is written as

$$
C_{t}=\left[\begin{array}{cc}
\alpha m_{1}+\frac{48 \beta k}{5} & -\frac{42 k \beta}{5} \\
-\frac{42 k \beta}{5} & \alpha m_{2}+\frac{48 \beta k}{5}
\end{array}\right]
$$

The dynamic equation after static-dynamic condensation would be

$$
M_{t} \ddot{u}+C_{t} \dot{u}+K_{t} u=-M_{t} I \ddot{u}_{0},
$$

where $M_{t}$ is the mass matrix, $M_{t}=\left[\begin{array}{ll}{ }^{m_{1}} & \\ & m_{2}\end{array}\right], u$, $\dot{u}$, and $\ddot{u}$ are the translational displacement, translational velocity, and translational acceleration, respectively. $I$ is a diagonal matrix defined; $\ddot{u}_{0}$ is the acceleration of applying excitation.
The state equation and observation equation can be derived according to the dynamic equation (16). The measured signals are the acceleration, $\ddot{u}_{1}$ and $\ddot{u}_{2}$. Hence, the state equation is

$$
\frac{d}{d_{t}}\left(\begin{array}{l}
u \\
\dot{u}
\end{array}\right)=\left[\begin{array}{cc}
0 & I \\
-M_{t}^{-1} K_{t} & -M_{t}^{-1} C_{t}
\end{array}\right]\left(\begin{array}{l}
u \\
\dot{u}
\end{array}\right)+\left(\begin{array}{c}
0 \\
-I
\end{array}\right) \ddot{u}_{0} .
$$

The stiffness $k$ and the damping parameters $\alpha$ and $\beta$ in the equation are regarded as the other three states of the system. Thus, all of the seven state arguments include $u_{1,0}$ which is the translational displacement of $m_{1}$ to foundation, $u_{2,0}$ which is the translational displacement of $m_{2}$ to foundation, $u_{3,0}$ which is the translational velocity of $m_{1}$ to foundation, $u_{4,0}$ which is 


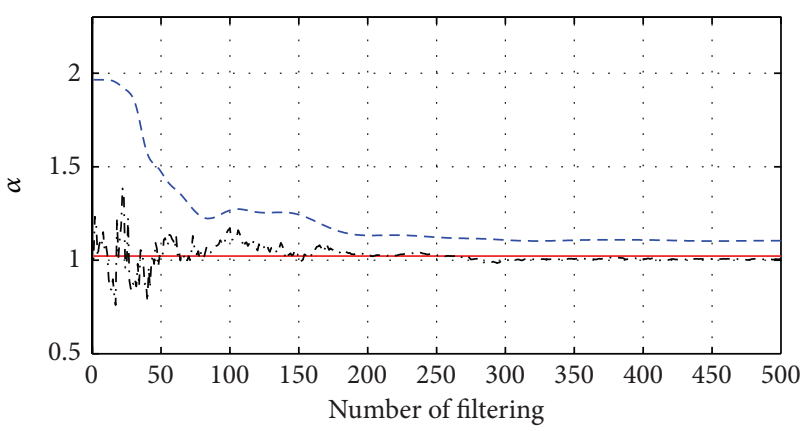

- Actual $\alpha$ in case 1

- _ - Identified $\alpha$ with no noise in case 1

. . . Identified $\alpha$ with noise in case 1

(a)

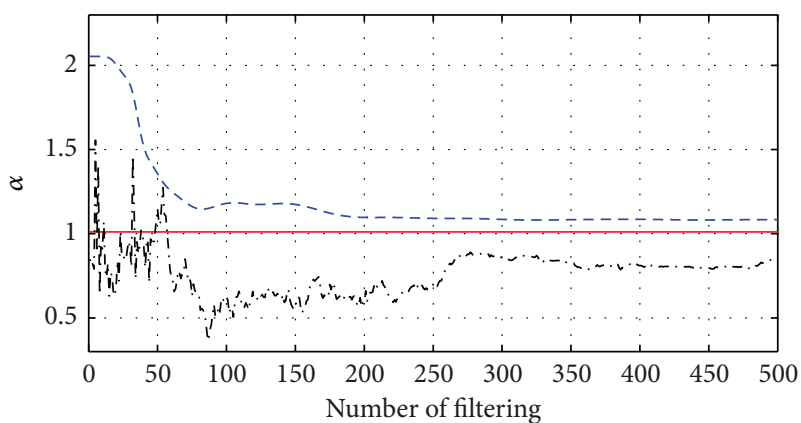

- Actual $\alpha$ in case 3

- - - Identified $\alpha$ with no noise in case 3

. . . Identified $\alpha$ with noise in case 3

(c)

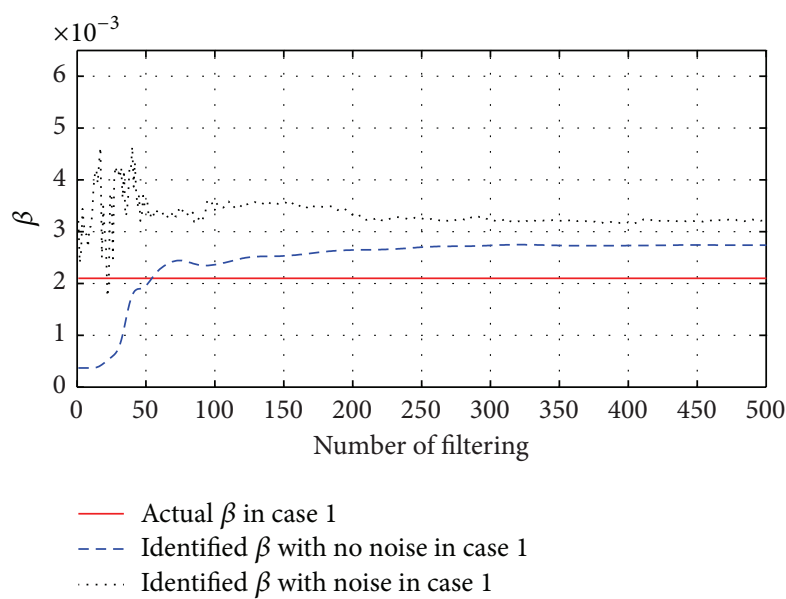

(b)

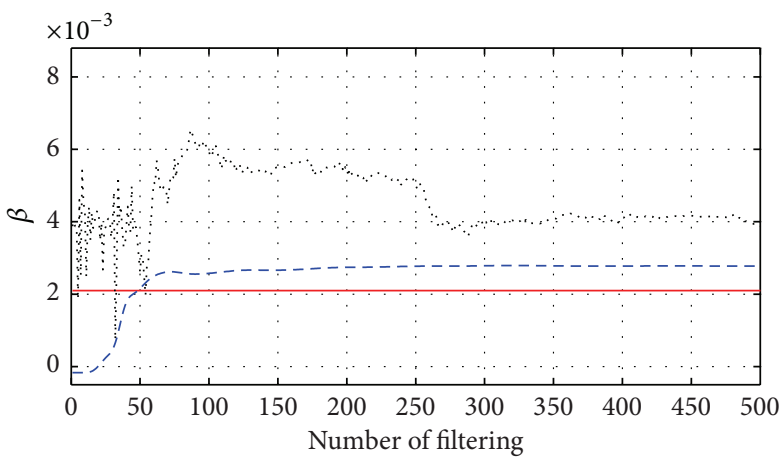

_ Actual $\beta$ in case 3

- - - Identified $\beta$ with no noise in case 3

..... Identified $\beta$ with noise in case 3

(d)

FIGURE 6: Identification results of $\alpha$ and $\beta$ in case 1 and case 3 under continuous excitation.

the translational velocity of $m_{2}$ to foundation, $u_{5}=k, u_{6}=\alpha$, and $u_{7}=\beta$. The system state equation is expressed as

$$
\begin{gathered}
\dot{u}_{1,0}=f_{1}=u_{3,0}, \\
\dot{u}_{2,0}=f_{2}=u_{4,0}, \\
\dot{u}_{3,0}=f_{3}=\frac{u_{5}\left(42 u_{2,0}-48 u_{1,0}\right)}{5 m_{1}} \\
\dot{u}_{4,0}=f_{4}=\frac{\left(5 u_{6} m_{1}+48 u_{7} u_{5} u_{3,0}-42 u_{7} u_{5} u_{4,0}\right)}{5 m_{1}}-\ddot{u}_{0}, \\
-\frac{\left(5 u_{6} m_{2}+48 u_{7} u_{5} u_{4,0}-48 u_{2,0}\right)}{5 m_{2}} \\
\dot{u}_{5}=\dot{u}_{6}=\dot{u}_{7}=0 .
\end{gathered}
$$

The observation equation is given by

$$
\begin{aligned}
Z_{1}=h_{1}= & \frac{u_{5}\left(42 u_{2,0}-48 u_{1,0}\right)}{5 m_{1}} \\
& -\frac{\left(5 u_{6} m_{1}+48 u_{7} u_{5} u_{3,0}-42 u_{7} u_{5} u_{4,0}\right)}{5 m_{1}} \\
Z_{2}=h_{2}= & \frac{u_{5}\left(42 u_{1,0}-48 u_{2,0}\right)}{5 m_{2}} \\
& -\frac{\left(5 u_{6} m_{2}+48 u_{7} u_{5} u_{4,0}-42 u_{7} u_{5} u_{3,0}\right)}{5 m_{2}} .
\end{aligned}
$$

According to (18)-(19), the state transition matrix of EKF equations can be obtained. Substituting the state equation and the observation equation into EKF equations with the reasonable sampling rate and calculating with iterative method, then the stiffness parameters and damping coefficient can be identified. Meanwhile, the velocity and the displacement can be estimated also. 


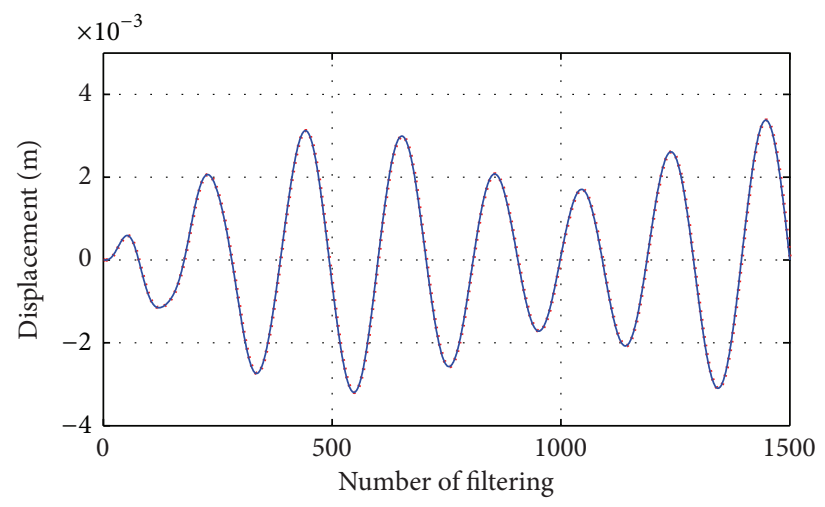

..... Estimated displacement with no noise — Actual displacement

(a)

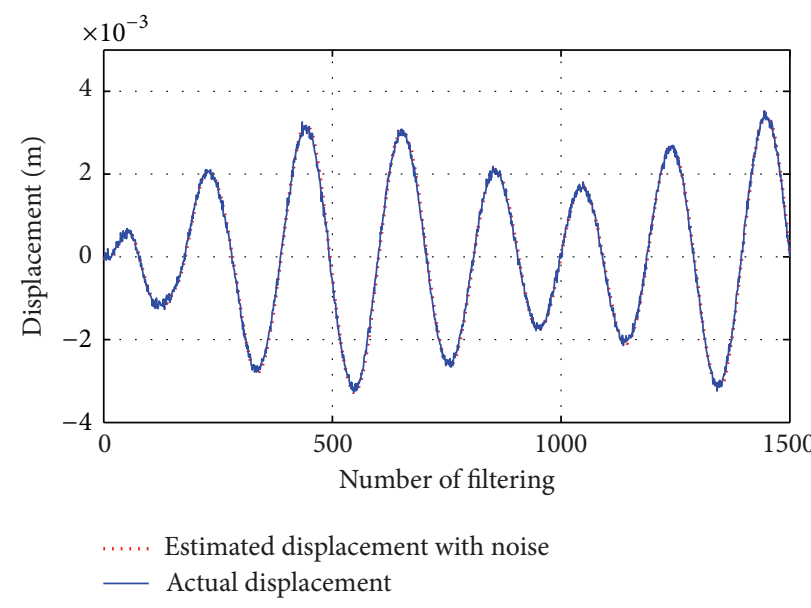

(c)

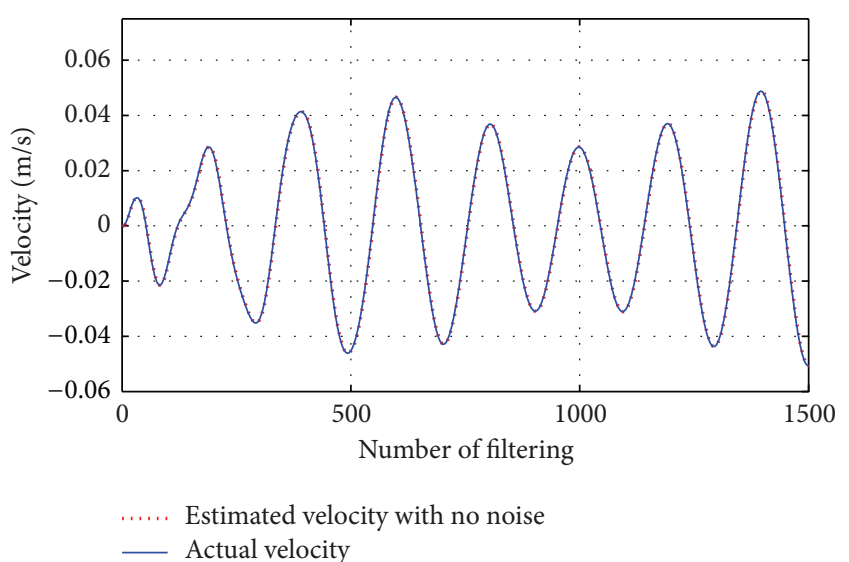

(b)

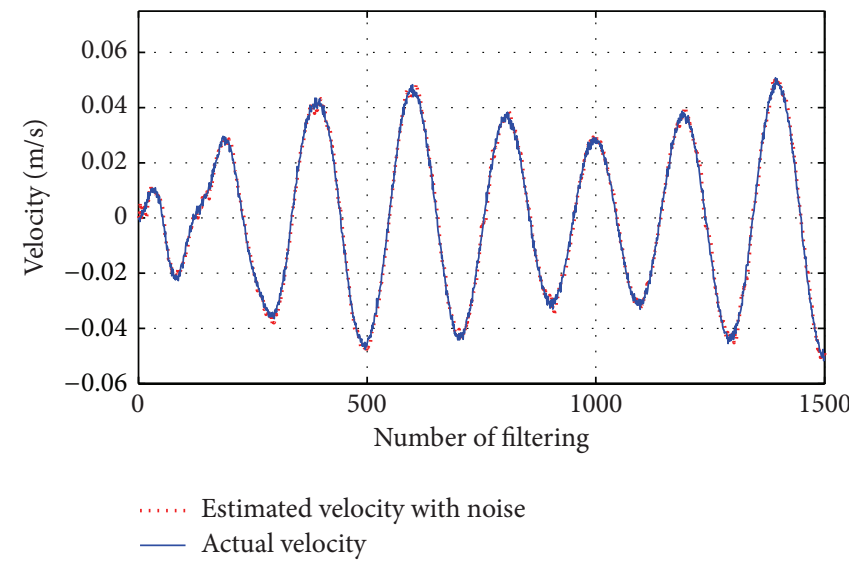

(d)

FIgURE 7: Actual and estimated response of point 1 in case 3 under continuous excitation.

\section{Excitation Generated by Impact Action}

The complete input and output signals are required for EKF; then the physical parameters are identified by the iteration and fitting. However, due to the interference by environmental factors, the excitation is difficult to be measured accurately in practice. Moreover, the continuous excitation has the disadvantages such as weak operability and worse convenience. If the bridge health monitoring is carried out under operational modal analysis when the ambient vibration is viewed as random excitations, the excitations on different locations are more difficult to be collected. The current solutions for obtaining the incomplete excitation signals mainly include assuming the known excitation or getting all the excitations according to the known time-history responses and the part of excitations, but these two methods are both inexact and defective if the number of the unknown input and output is large.

In the actual bridge inspection, the impact excitation generated by a hammer action or vehicle bumping is adopted in priority for the convenience, efficiency, and simplicity, and the bridge will behave as free vibration after the instantaneous impact is applied. Drop hammer and sledge hammer are two types of devices are often used in the test. An adjustable heavy moving mass drops from an adjustable height and the load cell with a medium polyurethane impact tip provides an impact on the surface of the deck [24]. While there are many variations in both size and force level, the hammers allow for modification of impact conditions through the use of impact tips with different stiffness. Because the impact carriage bounces off the bridge deck, several impacts can occur. The rebound control system aims to stop these multiple impacts and consists of a brake system activated by a control system that tracks the position of the impact carriage.

In practical monitoring, the acceleration responses of different locations are measured by accelerometers, and the sampling frequency should be properly chosen. Then the acceleration signals are processed by Fourier transform and the results in frequency domain are integrated. After the corresponding results are processed by inverse Fourier transform, the velocity signals are obtained. The displacement signals can be calculated by the same method. 


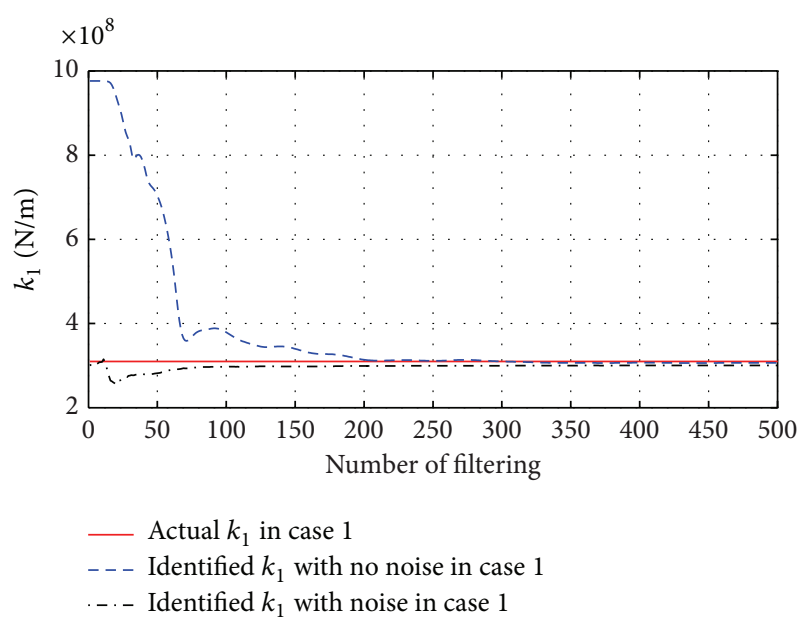

(a)

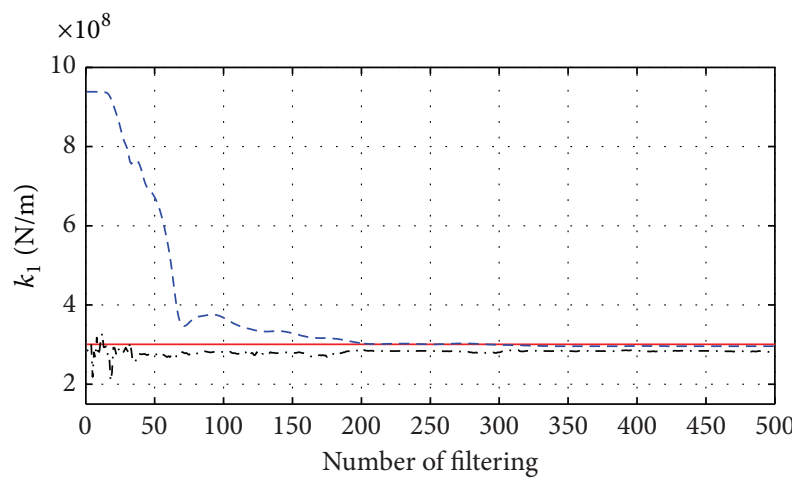

- Actual $k_{1}$ in case 2

- - - Identified $k_{1}$ with no noise in case 2

... Identified $k_{1}$ with noise in case 2

(c)

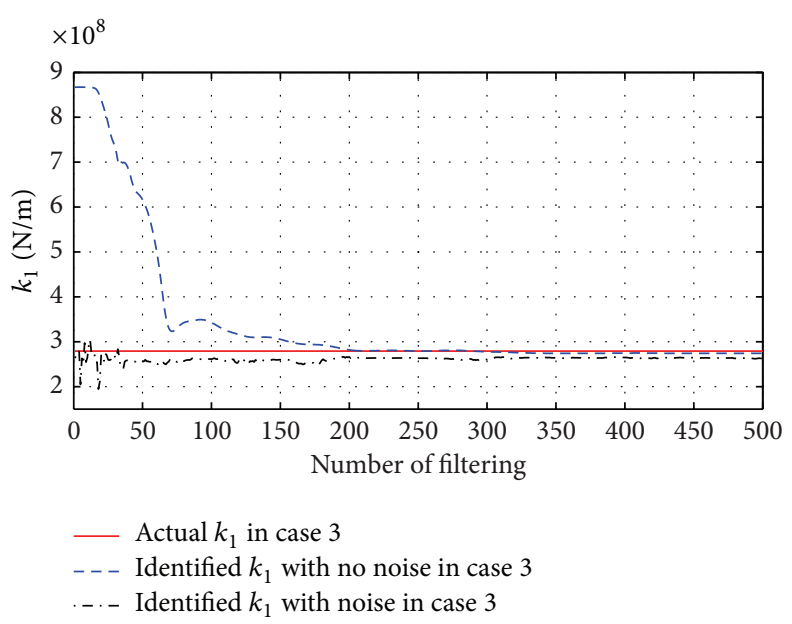

(b)

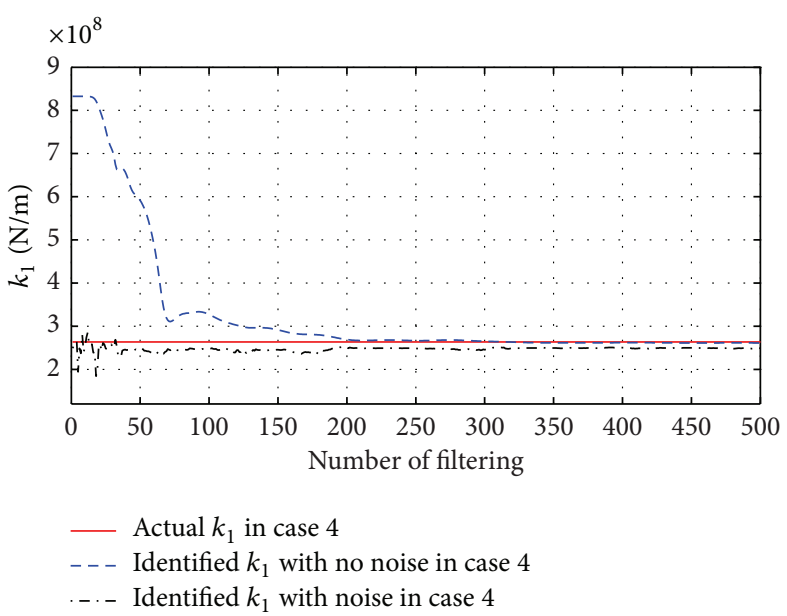

(d)

FIGURE 8: Identification results of $k_{1}$ in case 1 to case 4 under free vibration.

Hence, it is possible to obtain the time-history response of free vibration by means of only one or limited impact excitations are imposed on the specific locations on the deck, and the parameter can be identified by EKF as the case using continuous excitation. This method is convenient and has high signal-to-noise ratio because the amplitude of the pulse is greater than the amplitude of ambient vibration or normal exciters. Thus, the problem about incomplete excitations is solved by simplifying the excitation ingeniously but not using complex algorithms such as inversion and fitting.

In the equations of EKF, the pulse excitation is transient so the force is measured and estimated in the initial time, and then the input values in the state equations are zero. When the excitation is generated by vehicle bumping, the mass of the engineering truck is large and the mass should be considered as the additional mass of the bridge once the truck landed, so the corresponding mass matrix is modified. When the excitation is generated by power hammers, the mass of the hammers can be ignored. It is verified that the loading mode nearly does not affect the identification accuracy in Kalman filtering; thus, the technique for obtaining vibration signals by pulse excitation is feasible and especially suitable for online damage detection and health monitoring.

\section{Damage Identification for Three-Span Continuous Girder Bridge}

In order to verify the effect of damage detection method based on static-dynamic condensation and extended Kalman filtering proposed in this paper, a three-span continuous girder bridge is analyzed as an example; the results for different locations and damage degrees are discussed, and the effect of noise is also considered. The three-span prestressed concrete variable box sections continuous girder bridge is shown in Figure 3. The length of the bridge is $150 \mathrm{~m}$; the cross section bending moment of inertia in the middle of main span is $10 \mathrm{~m}^{4}$; the cross section bending moment of inertia in the end support of bridge is $12.5 \mathrm{~m}^{4}$ and $87.5 \mathrm{~m}^{4}$ for the support in middle. The elasticity modulus of reinforced concrete $E$ is $3.1 \times 10^{10} \mathrm{~N} / \mathrm{m}^{2}$, and the mass of the total girderis $4.77 \times 10^{6} \mathrm{~kg}$. The bridge can be simplified as the model shown 


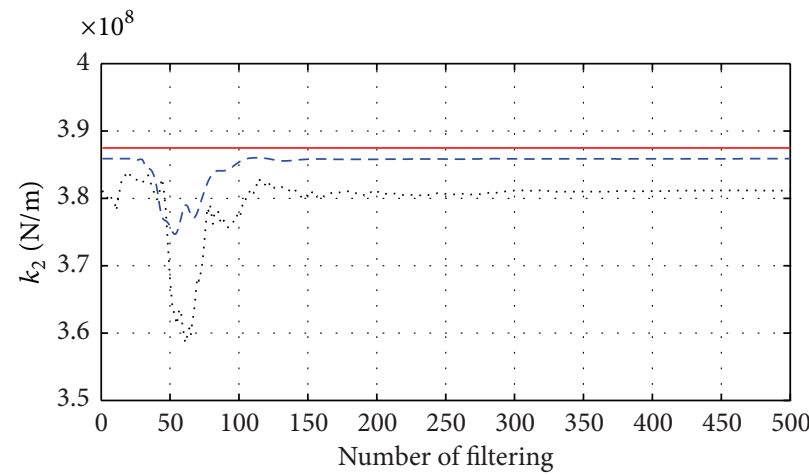

- Actual $k_{2}$ in case 1

- - Identified $k_{2}$ with no noise in case 1

..... Identified $k_{2}$ with noise in case 1

(a)

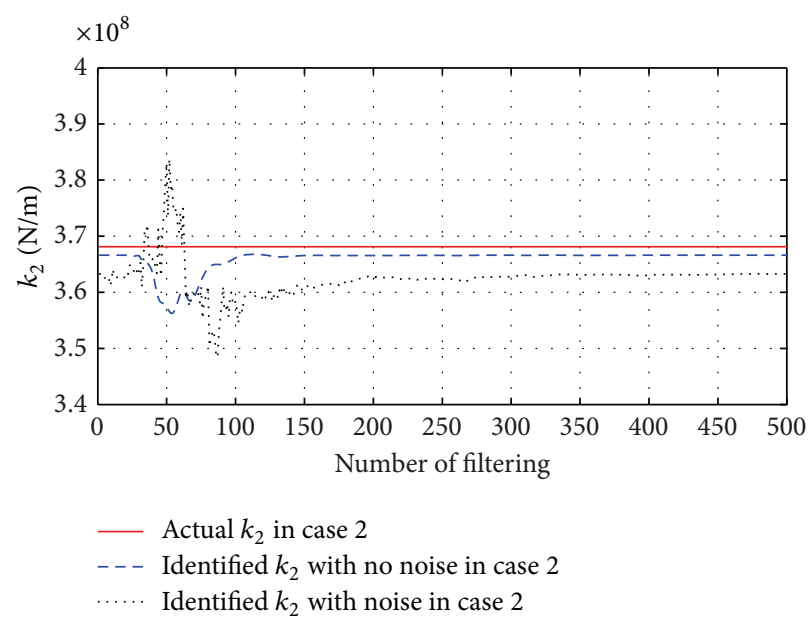

(c)

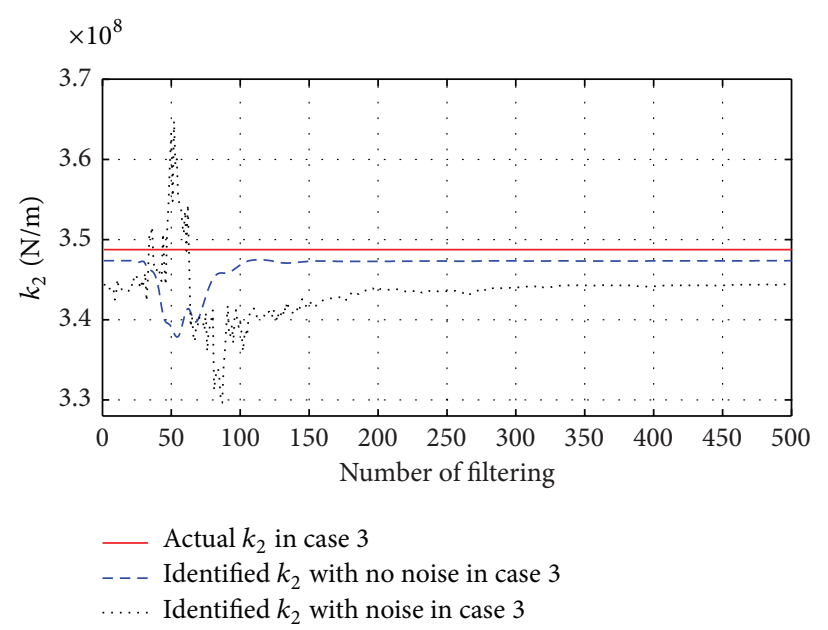

(b)

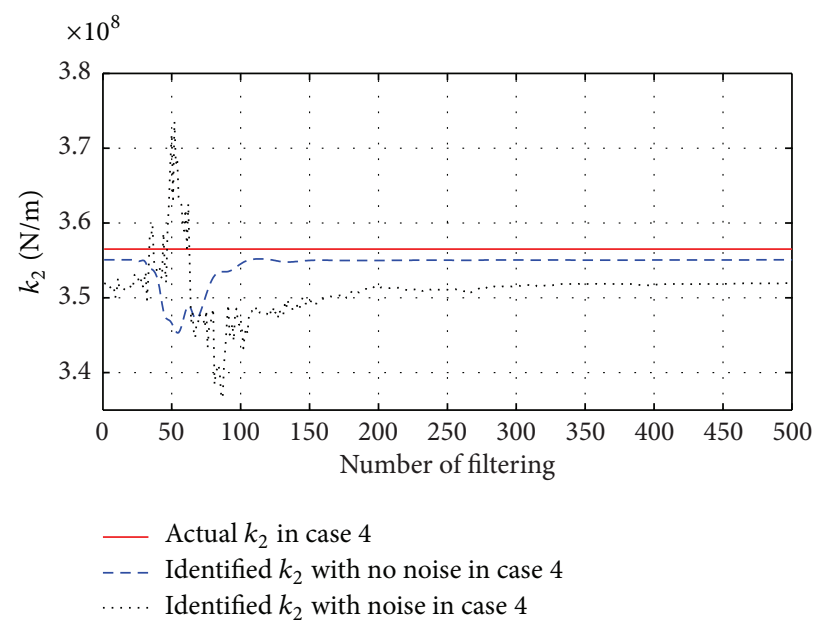

(d)

FIgURE 9: Identification results of $k_{2}$ in case 1 to case 4 under free vibration.

in Figure 4, which is divided into 15 elements. The length of each element $l$ is $10 \mathrm{~m}$. The stiffness of each element is given as $k=E I / l^{3}$. The cross section at bearings and midspan is the weakest according to the engineering investigation and experience, so the stiffness $k_{1}$ of element 6-7 and $k_{2}$ of element 13-1 are assumed as the identified parameters (the actual $k_{1}$ and $k_{2}$ are $3.1 \times 10^{8} \mathrm{~N} / \mathrm{m}$ and $3.875 \times 10^{8} \mathrm{~N} / \mathrm{m}$, resp.). In addition, Rayleigh damping parameters $\alpha, \beta$ are also identified. The damping ratios under different damage conditions are all assumed to be 0.05 . According to the equation as follows:

$$
\left(\begin{array}{l}
\alpha \\
\beta
\end{array}\right)=\frac{2 \xi}{\omega_{1}+\omega_{3}}\left(\begin{array}{c}
\omega_{1} \omega_{3} \\
1
\end{array}\right)
$$

where $\omega_{1}$ and $\omega_{3}$ are the first- and third-order modal frequencies, $\xi$ is the damping ratio that assumed to be 0.05 , hence, the damping coefficients $\alpha$ and $\beta$ can be calculated and the damping coefficient $\alpha$ is $1.022,1.018,1.011$, and 1.007 for the four cases, respectively, and $\beta$ is 0.002 . Considering the interference of the noise, the stiffness with different damage degrees is identified under the condition that every mass point is continuously excited or only one point is excited by an instantaneous impact load. The sampling frequency of the signals is $500 \mathrm{~Hz}$.

First, the damage cases are detected under the continuous excitation to each mass point. The expression form of the excitation is $y=0.4 \sin (4 \pi t+9.96)+0.5 \cos (5 \pi t+20)$. The detailed results are shown in Table 1 and Figures 5, 6, and 7. It is evident that the response history about the displacement and the velocity is estimated accurately and identification accuracy of the stiffness and the damping parameters meet the actual requirement.

In general, if the initial covariance of the state vectors is larger, the rate of convergence is higher, but the identification accuracy is not stable and the final results maybe inexact. For the signals without noise, the identification will converge quickly, and the convergence is worse for the signals with noise. Hence, the initial covariance is set smaller for the signals without noise in order to obtain more stable solutions. On the contrary, the initial covariance is larger for the signals with noise to ensure that the identification converges quickly and the divergence is avoided, but the final accuracy is lower. 


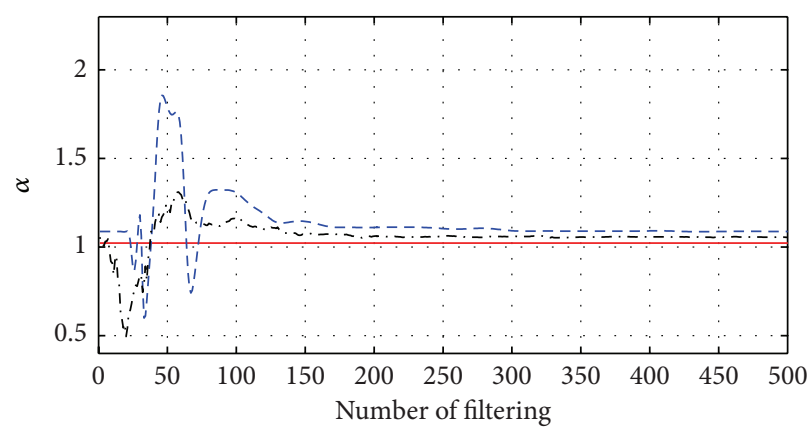

- Actual $\alpha$ in case 1

- - - Identified $\alpha$ with no noise in case 1

...- Identified $\alpha$ with noise in case 1

(a)

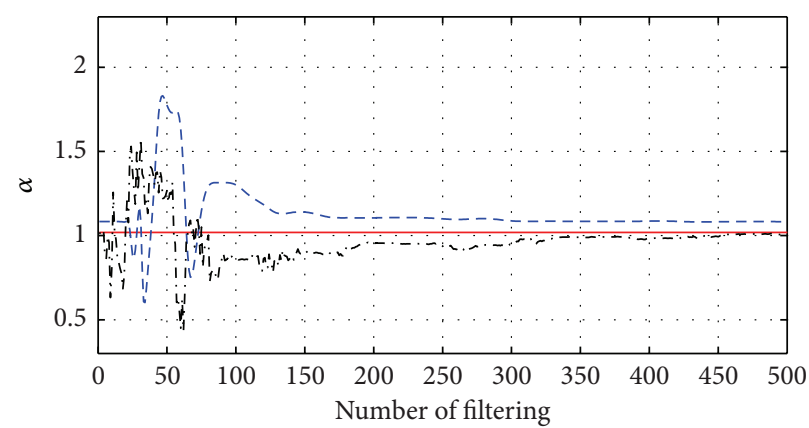

- Actual $\alpha$ in case 2

- - - Identified $\alpha$ with no noise in case 2

. . . - Identified $\alpha$ with noise in case 2

(c)

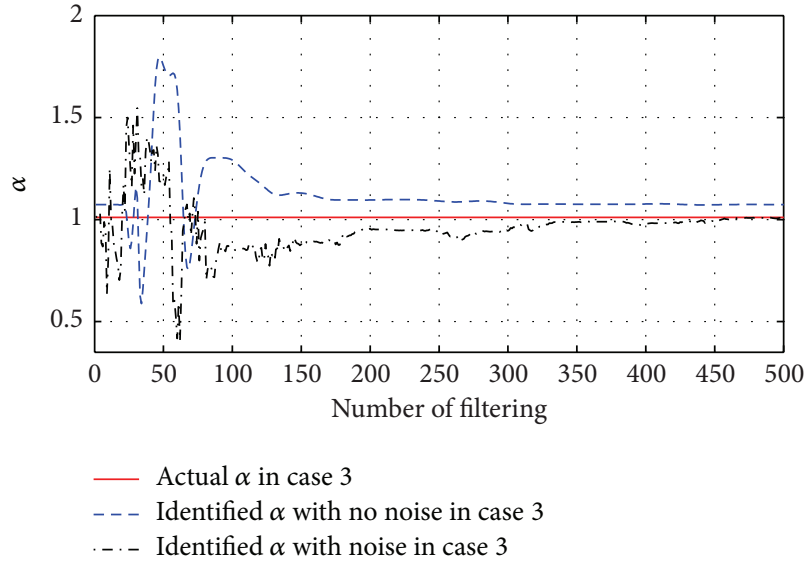

(b)

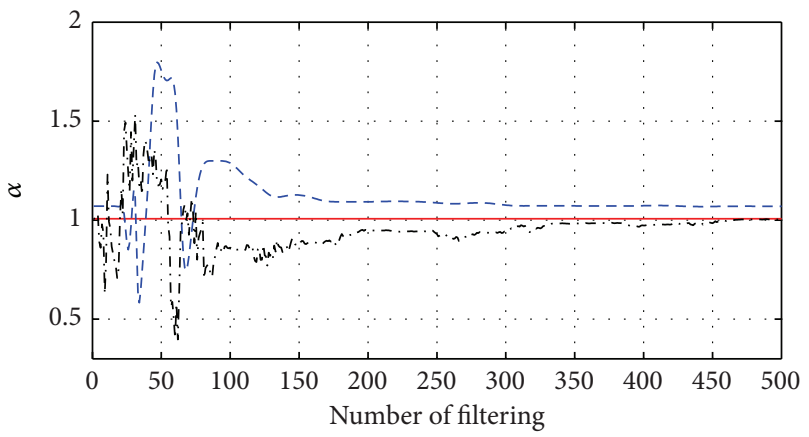

- Actual $\alpha$ in case 4

- - - Identified $\alpha$ with no noise in case 4

. . . Identified $\alpha$ with noise in case 4

(d)

Figure 10: Identification results of $\alpha$ in case 1 to case 4 under free vibration.

TABLE 1: The parameter identification results under continuous excitation.

\begin{tabular}{|c|c|c|c|c|c|c|c|c|c|}
\hline \multirow{2}{*}{ Case } & \multirow{2}{*}{$k_{1}, k_{2}$ damage } & \multicolumn{4}{|c|}{ No noise } & \multicolumn{4}{|c|}{$5 \%$ noise } \\
\hline & & $k_{1}(\mathrm{~N} / \mathrm{m})$ (error) & $k_{2}(\mathrm{~N} / \mathrm{m})$ (error) & $\alpha$ & $\beta$ & $k_{1}(\mathrm{~N} / \mathrm{m})$ (error) & $k_{2}(\mathrm{~N} / \mathrm{m})$ (error) & $\alpha$ & $\beta$ \\
\hline 1 & $k_{1} k_{2}$ intact & $3.10 \times 10^{8}(-0.03 \%)$ & $3.87 \times 10^{8}(-0.18 \%)$ & 1.10 & $2.72 \times 10^{-3}$ & $3.06 \times 10^{8}(1.3 \%)$ & $3.86 \times 10^{8}(0.5 \%)$ & 1.00 & $3.20 \times 10^{-3}$ \\
\hline 2 & $\begin{array}{l}k_{1} 3 \% \\
k_{2} 5 \%\end{array}$ & $3.00 \times 10^{8}(-0.1 \%)$ & $3.56 \times 10^{8}(-3.1 \%)$ & 1.09 & $2.83 \times 10^{-3}$ & $2.93 \times 10^{8}(2.6 \%)$ & $3.65 \times 10^{8}(0.9 \%)$ & 0.85 & $3.90 \times 10^{-3}$ \\
\hline 3 & $\begin{array}{l}k_{1} 10 \% \\
k_{2} 10 \%\end{array}$ & $2.79 \times 10^{8}(0.08 \%)$ & $3.48 \times 10^{8}(0.16 \%)$ & 1.08 & $2.77 \times 10^{-3}$ & $2.73 \times 10^{8}(2.1 \%)$ & $3.45 \times 10^{8}(1.1 \%)$ & 0.84 & $3.90 \times 10^{-3}$ \\
\hline 4 & $\begin{array}{l}k_{1} 15 \% \\
k_{2} 8 \%\end{array}$ & $2.63 \times 10^{8}(-0.07 \%)$ & $3.56 \times 10^{8}(-0.17 \%)$ & 1.08 & $2.75 \times 10^{-3}$ & $2.61 \times 10^{8}(0.95 \%)$ & $3.57 \times 10^{8}(0.14 \%)$ & 1.10 & $2.75 \times 10^{-3}$ \\
\hline
\end{tabular}

Thus, at the beginning of filtering, the identification accuracy of the stiffness in case with noise is significantly higher than that in case without noise.

In case of no noise, the difference between the identification value and real value of stiffness, $k_{1}$ and $k_{2}$, is very slight and the errors can be ignored. The noise-signal ratio is defined as the ratio of the noise amplitude to the maximum response of the structure. When the noise-signal ratio is $5 \%$, the identification accuracy decreased slightly but it still meets the accuracy requirements. Since the Rayleigh damping parameters $\alpha$ and $\beta$ are the values with small order of magnitude, the identification accuracy is lower but acceptable.

The sampling rate of the signals is 500 and it can be seen from the results that the identification generally converged after 500 steps, so the damage identification finished only in one second and the accuracy is enough. Hence, the operation time is short.

In order to reduce the influence to the identified location when the bridge is subjected to impact load, the instantaneous load is separately applied on mass point 11, the damage detection is carried out, and the damage identification results 


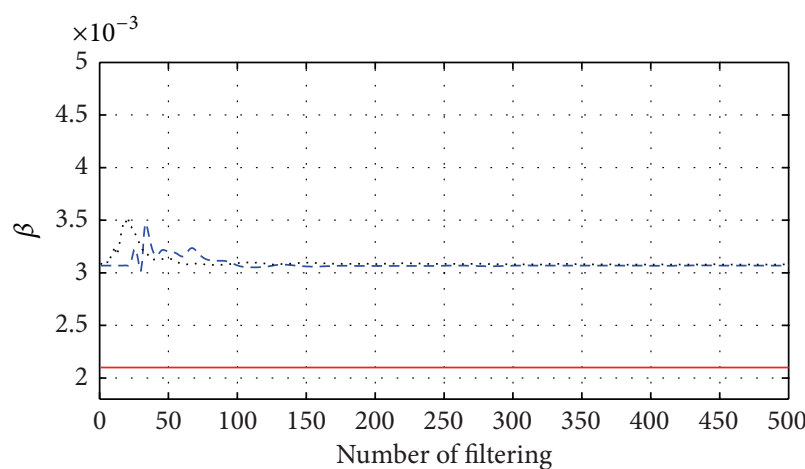

- Actual $\beta$ in case 1

- - - Identified $\beta$ with no noise in case 1

..... Identified $\beta$ with noise in case 1

(a)

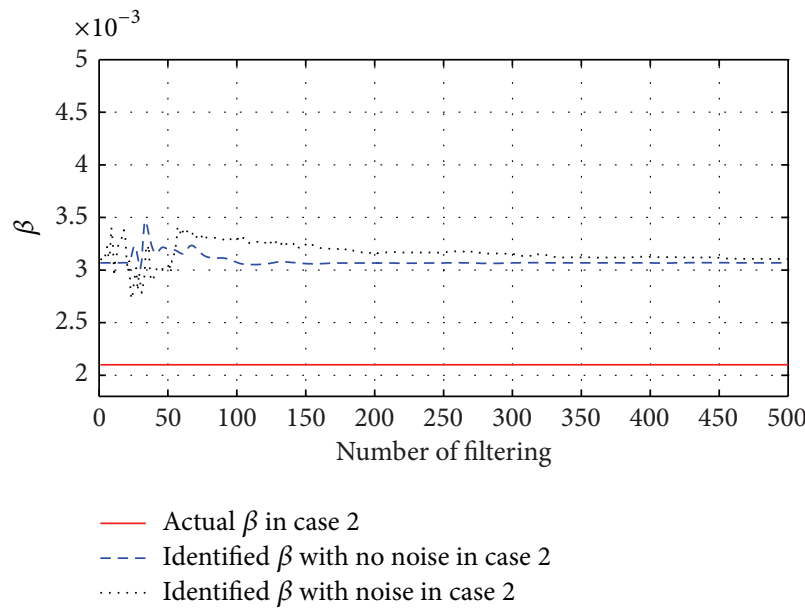

(c)

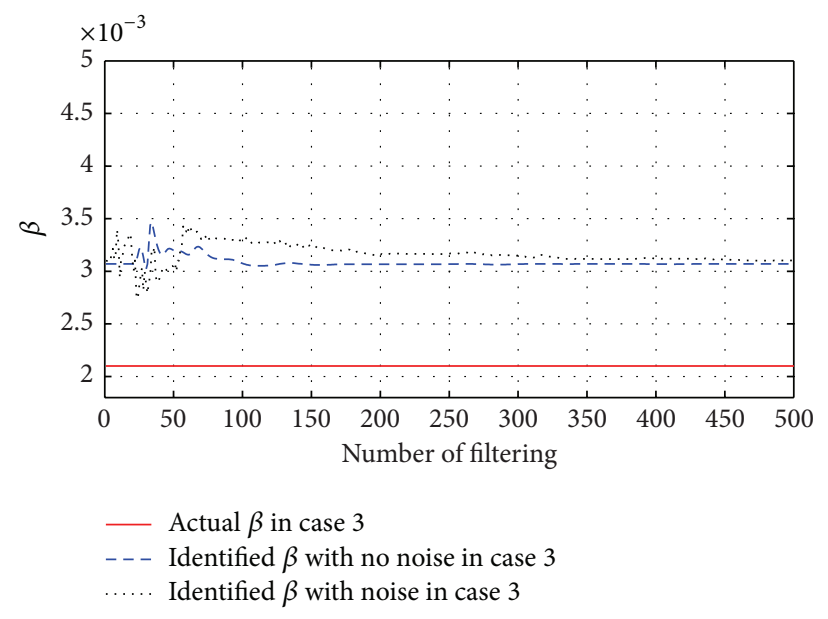

(b)

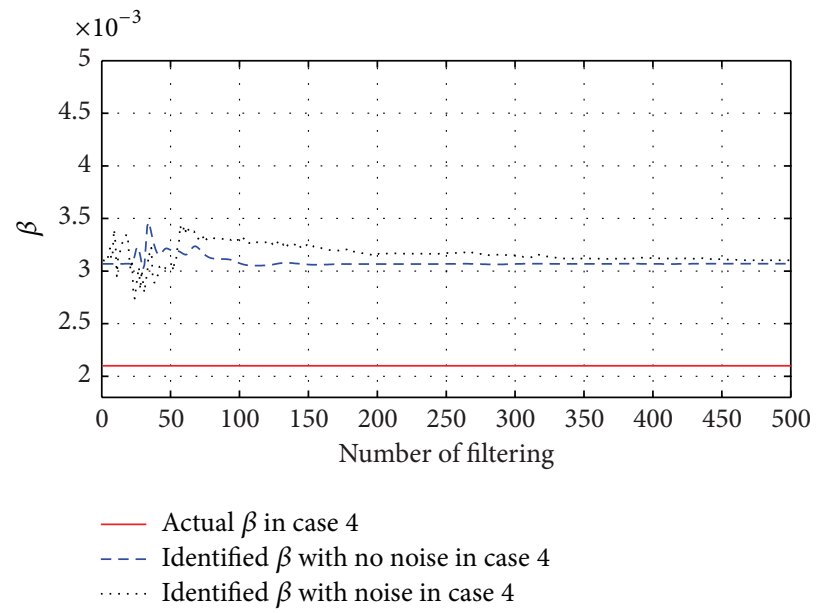

(d)

Figure 11: Identification results of $\beta$ in case 1 to case 4 under free vibration.

TABLE 2: Parameter identification results under free vibration.

\begin{tabular}{|c|c|c|c|c|c|c|c|c|c|}
\hline \multirow{2}{*}{ Case } & \multirow{2}{*}{$k_{1} k_{2}$ damage } & \multicolumn{4}{|c|}{ No noise } & \multicolumn{4}{|c|}{$5 \%$ noise } \\
\hline & & $k_{1}(\mathrm{~N} / \mathrm{m})$ (error) & $k_{2}(\mathrm{~N} / \mathrm{m})$ (error) & $\alpha$ & $\beta$ & $k_{1}(\mathrm{~N} / \mathrm{m})$ (error) & $k_{2}(\mathrm{~N} / \mathrm{m})$ (error) & $\alpha$ & $\beta$ \\
\hline 1 & $k_{1} k_{2}$ intact & $3.063 \times 10^{8}(1.2 \%)$ & $3.858 \times 10^{8}(0.44 \%)$ & 1.087 & $3.06 \times 10^{-3}$ & $3.01 \times 10^{8}(2.9 \%)$ & $3.81 \times 10^{8}(1.6 \%)$ & 1.056 & $3.00 \times 10^{-3}$ \\
\hline 2 & $\begin{array}{l}k_{1} 3 \% \\
k_{2} 5 \%\end{array}$ & $2.958 \times 10^{8}(1.6 \%)$ & $3.666 \times 10^{8}(0.41 \%)$ & 1.083 & $3.068 \times 10^{-3}$ & $2.82 \times 10^{8}(6.6 \%)$ & $3.63 \times 10^{8}(1.3 \%)$ & 1.008 & $3.10 \times 10^{-3}$ \\
\hline 3 & $\begin{array}{l}k_{1} 10 \% \\
k_{2} 10 \%\end{array}$ & $2.741 \times 10^{8}(1.7 \%)$ & $3.474 \times 10^{8}(0.13 \%)$ & 1.074 & $3.069 \times 10^{-3}$ & $2.64 \times 10^{8}(5.3 \%)$ & $3.45 \times 10^{8}(0.8 \%)$ & 1.008 & $3.10 \times 10^{-3}$ \\
\hline 4 & $\begin{array}{c}k_{1} 15 \% \\
k_{2} 8 \%\end{array}$ & $2.612 \times 10^{8}(2.3 \%)$ & $3.550 \times 10^{8}(0.42 \%)$ & 1.069 & $3.069 \times 10^{-3}$ & $2.49 \times 10^{8}(6.1 \%)$ & $3.52 \times 10^{8}(1.2 \%)$ & 1.002 & $3.10 \times 10^{-3}$ \\
\hline
\end{tabular}

are shown in Table 2 and Figures 8, 9, 10, 11, and 12. It is verified that the state parameter including the velocity and the displacement is estimated accurately under free vibration. Although the identification accuracy of stiffness is less than the results of the continuous excitation, it also can meet the requirement. There also exit certain errors for the identification of Rayleigh damping coefficients $\alpha$ and $\beta$, but the error is stable and acceptable.
In order to prove the noise immunity of the damage detection method, $20 \%$ noise is added in the signals in case 3 under the free vibration and the results are shown in Figures 13 and 14. The stiffness is estimated accurately even though the noise-signal ratio is high, but the error for $\alpha$ and $\beta$ is evident. In general, the damage detection for locations and degree is available for the condition with strong noise even under free vibration. 


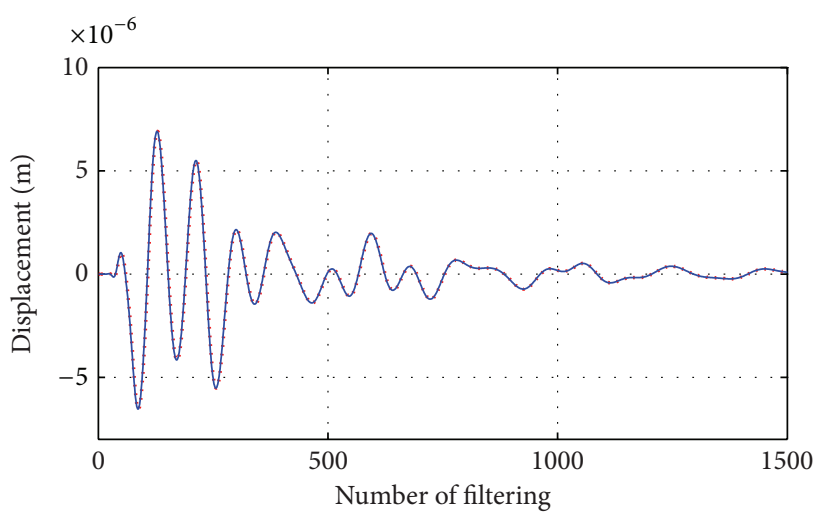

..... Estimated displacement with no noise — Actual displacement

(a)

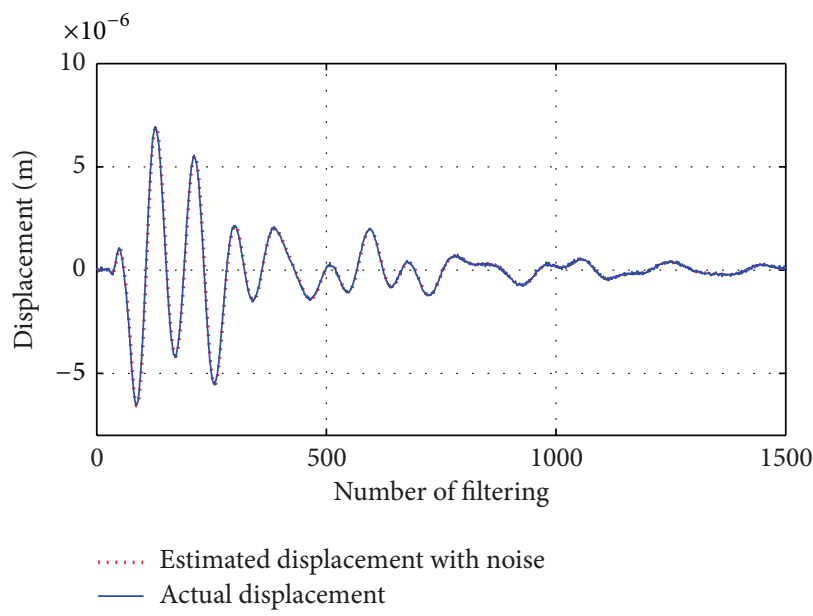

(c)

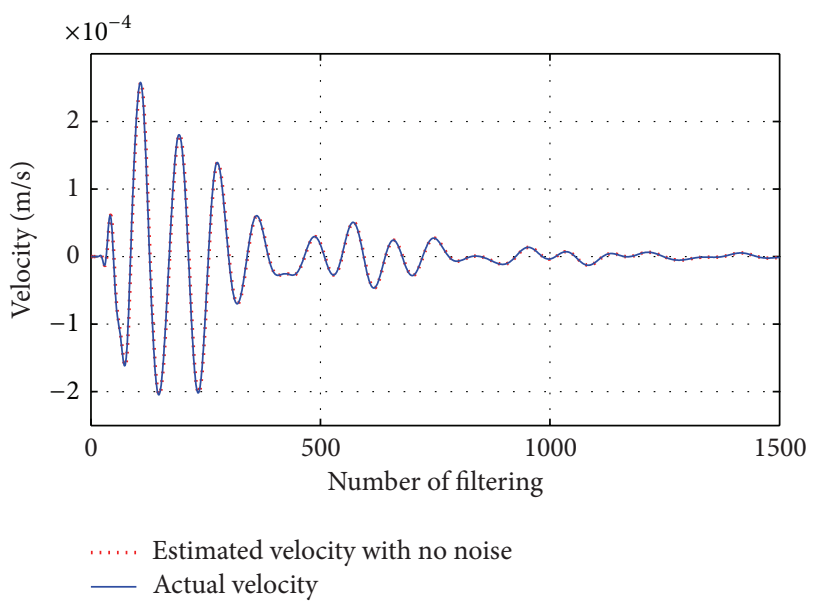

(b)

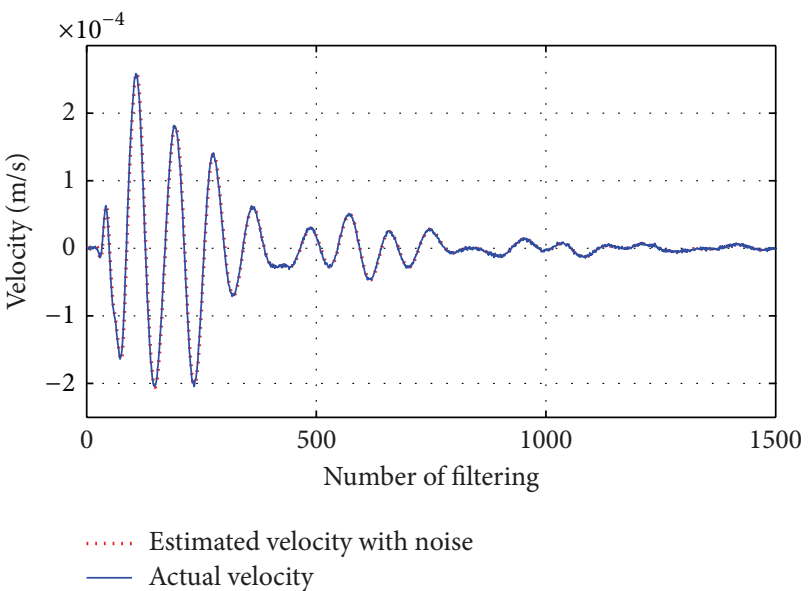

(d)

Figure 12: Actual and estimated response of point 1 in case 3 under free vibration.

\section{Conclusions}

A damage detection method based on static-dynamic condensation and extended Kalman filtering is presented. In order to solve the problems on complete inputs and outputs, the derivation about the static-dynamic condensation is given and the degrees of freedom about rotational can be eliminated. Furthermore, the impact load applied only on one position is advised since it is simple, rapid, and effective. The method is verified through an example for a threespan prestressed continuous bridge. The results show that the stiffness identification is accurate under multiple point which is actuated by trigonometric function excitation, and the accuracy is also acceptable for impact excitation only on one point. The damage detection method is insensitive to noise even though the noise-signal ratio is up to $20 \%$, so the method proposed in this paper can be applied in bridge fast inspection and health monitoring, and the operation time is short and the corresponding instruments for excitation and acquisition are portable. There exits certain error in identifications for Rayleigh damping factor, $\alpha$ and $\beta$; it is worth further study in particular.

The damage detection method will be improved in some aspects as follows. (1) Large computation occurs when the number of the elements in the structures is large; the modeling and the feasibility for more complex bridge will be studied in the future. The parallel computing technology will be applied to enhance the computational efficiency. (2) Damage detection experiment on the continuous girder bridge is needed to verify the conclusion in numerical simulations, and the environmental factors such as temperature and humidity should be considered. The modification for identified damping factor will be studied. (3) The effect of piers and the modal shapes in horizontal direction is ignored, the detection capacity for combined damage cases and the modal coupling will be considered further. 


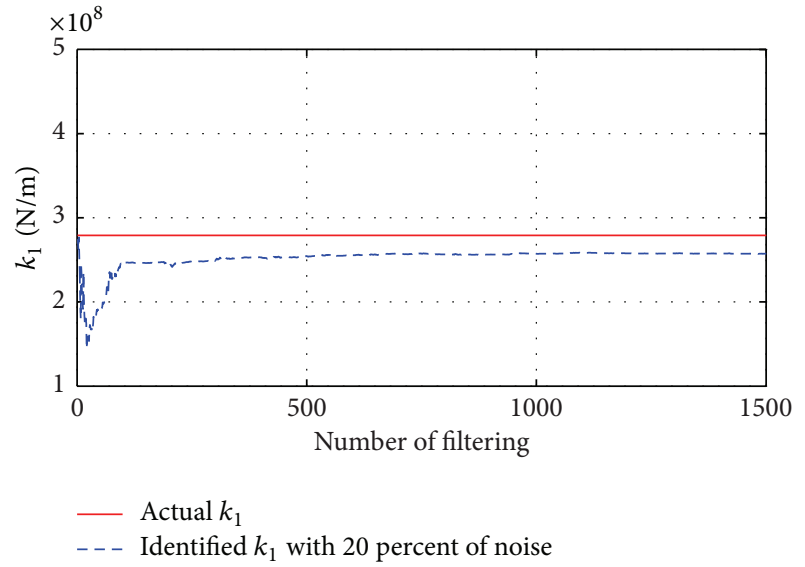

(a)

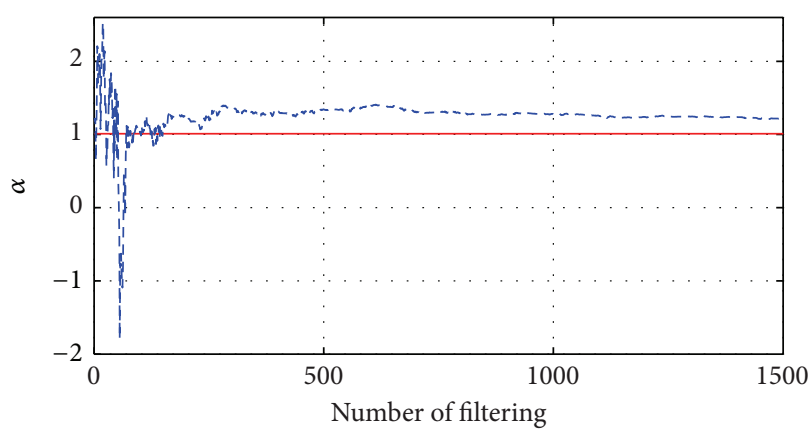

Actual $\alpha$

Identified $\alpha$ with 20 percent of noise

(c)

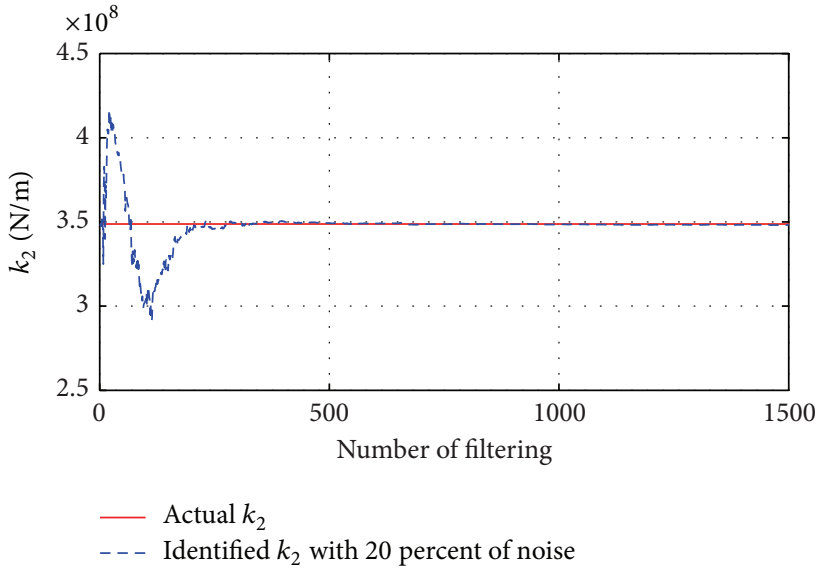

(b)

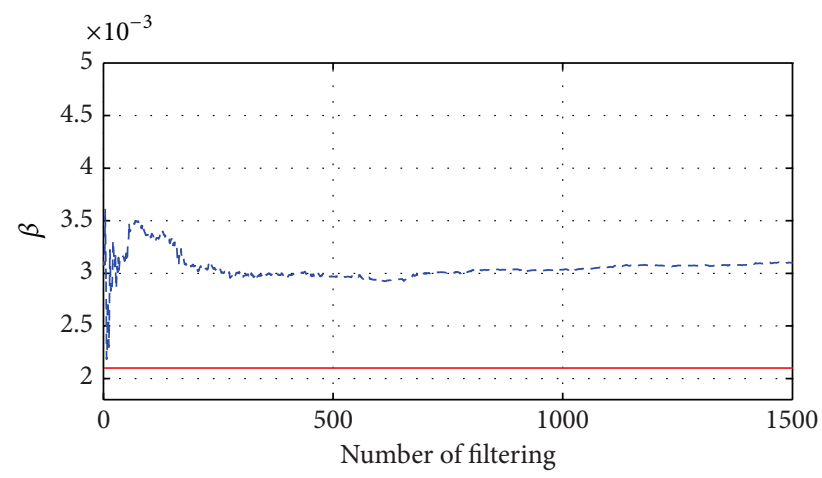

_ Actual $\beta$

- - Identified $\beta$ with 20 percent of noise

(d)

Figure 13: Results of $k_{1}, k_{2}, \alpha$, and $\beta$ with $20 \%$ noise under free vibration.

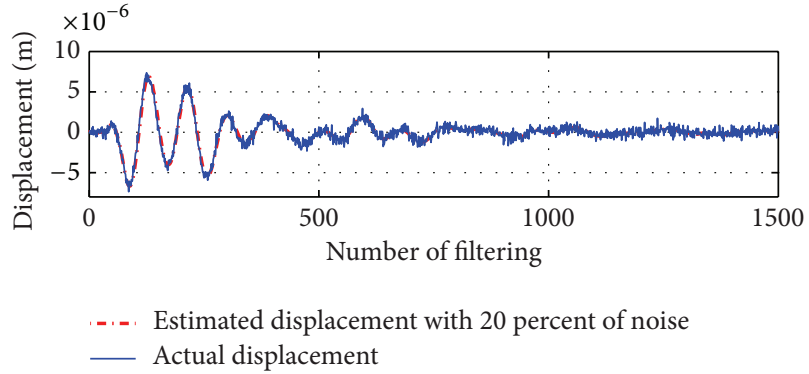

(a)

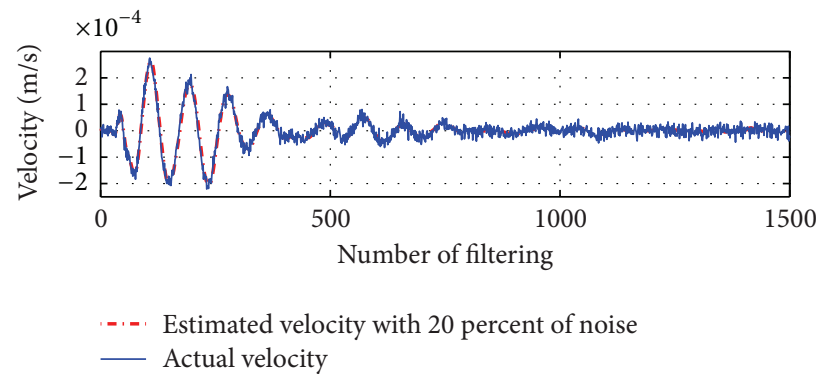

(b)

FIGURE 14: Actual and estimated response of point 1 with 20\% noise under free vibration.

\section{Conflict of Interests}

The authors declare that there is no conflict of interests regarding the publication of this paper.

\section{Acknowledgments}

This work is partially supported by the Natural Science Foundation of China under Grant nos. 51108009 and 50878010, Foundation of the Ministry of Education of China for
Outstanding Young Teachers in University under Grant no. 20111103120022, and Foundation of Beijing Key Lab of Earthquake Engineering and Structural Retrofit under Grant no. 2013TS02.

\section{References}

[1] Z. S. Wu, B. Xu, and T. Harad, "Review on structural health monitoring for infrastructures," Applied Mechanics, vol. 6, pp. 1043-1054, 2003. 
[2] S. W. Doebling, C. R. Farrar, and M. B. Prime, "A summary review of vibration-based damage identification methods," Shock and Vibration Digest, vol. 30, no. 2, pp. 91-105, 1998.

[3] H. Li, T. Yi, M. Gu, and L. Huo, "Evaluation of earthquakeinduced structural damages by wavelet transform," Progress in Natural Science, vol. 19, no. 4, pp. 461-470, 2009.

[4] C. K. Chui and G. Chen, Kalman Filtering with Real-Time Applications, Springer, Berlin, Germany, 3rd edition, 1998.

[5] D. Wang and A. Haldar, "Element-level system identification with unknown input," Journal of Engineering Mechanics, vol. 120, no. 1, pp. 159-176, 1994.

[6] D. Wang and A. Haldar, "System identification with limited observations and without input," Journal of Engineering Mechanics, vol. 123, no. 5, pp. 504-511, 1997.

[7] X. Ling and A. Haldar, "Element level system identification with unknown input with Rayleigh damping," Journal of Engineering Mechanics, vol. 130, no. 8, pp. 877-885, 2004.

[8] S. J. Ghosh, D. Roy, and C. S. Manohar, "New forms of extended Kalman filter via transversal linearization and applications to structural system identification," Computer Methods in Applied Mechanics and Engineering, vol. 196, no. 49-52, pp. 5063-5083, 2007.

[9] W. Meiliang and A. W. Smyth, "Application of the unscented Kalman filter for real-time nonlinear structural system identification," Structural Control and Health Monitoring, vol. 14, no. 7, pp. 971-990, 2007.

[10] J. N. Yang, S. Pan, and H. Huang, "An adaptive extended Kalman filter for structural damage identifications II: unknown inputs," Structural Control and Health Monitoring, vol. 14, no. 3, pp. 497521, 2007.

[11] J. N. Yang, S. Pan, and S. Lin, "Least-squares estimation with unknown excitations for damage identification of structures," Journal of Engineering Mechanics, vol. 133, no. 1, pp. 12-21, 2007.

[12] J. N. Yang, H. W. Huang, and S. L. Lin, "Sequential non-linear least-square estimation for damage identification of structures," International Journal of Non-Linear Mechanics, vol. 41, no. 1, pp. 124-140, 2006.

[13] Y. Lei, Y. Wu, and T. Li, "Identification of non-linear structural parameters under limited input and output measurements," International Journal of Non-Linear Mechanics, vol. 47, no. 10, pp. 1141-1146, 2012.

[14] Y. Lei, Y. Q. Jiang, and Z. Q. Xu, "Structural damage detection with limited input and output measurement signals," Mechanical Systems and Signal Processing, vol. 28, pp. 229-243, 2012.

[15] Y. Lei and Y. Q. Jiang, "A two-stage Kalman estimation approach for the identification of nonlinear structural parameters," Procedia Engineering, vol. 14, pp. 3088-3094, 2011.

[16] Y. Lei, C. Liu, Y. Q. Jiang, and Y. K. Mao, "Substructure based structural damage detection with limited input and output measurements," Smart Structures and Systems, vol. 12, no. 6, pp. 619-640, 2013.

[17] R. Tipireddy, H. A. Nasrellah, and C. S. Manohar, "A Kalman filter based strategy for linear structural system identification based on multiple static and dynamic test data," Probabilistic Engineering Mechanics, vol. 24, no. 1, pp. 60-74, 2009.

[18] F. Bakhtiari-Nejad, A. Rahai, and A. Esfandiari, "A structural damage detection method using static noisy data," Engineering Structures, vol. 27, no. 12, pp. 1784-1793, 2005.

[19] L. Zhou, S. Y. Wu, and J. N. Yang, "Experimental study of an adaptive extended Kalman filter for structural damage identification," Journal of Infrastructure Systems, vol. 14, no. 1, pp. $42-51,2008$.
[20] H. Huang, J. N. Yang, and L. Zhou, "Adaptive quadratic sumsquares error with unknown inputs for damage identification of structures," Structural Control and Health Monitoring, vol. 17, no. 4, pp. 404-426, 2010.

[21] M. Hoshiya and E. Saito, "Structural identification by extended Kalman filter," Journal of Engineering Mechanics, vol. 110, no. 12, pp. 1757-1770, 1984.

[22] T. H. Yi, H. N. Li, and X. Y. Zhao, "Noise smoothing for structural vibration test signals using an improved wavelet thresholding technique," Sensors, vol. 12, no. 8, pp. 11205-11220, 2012.

[23] A. K. Chopra, Dynamics of Structures Theory and Applications to Earthquakes Engineering, Prentice Hall, 4th edition, 2011.

[24] J. B. Prader, Rapid impact modal testing for bridge flexibility: towards objective evaluation of infrastructures [Ph.D. thesis], Drexel University, 2012. 


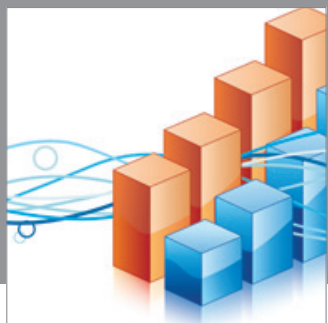

Advances in

Operations Research

mansans

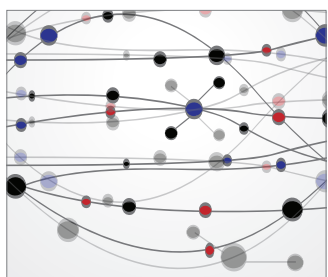

The Scientific World Journal
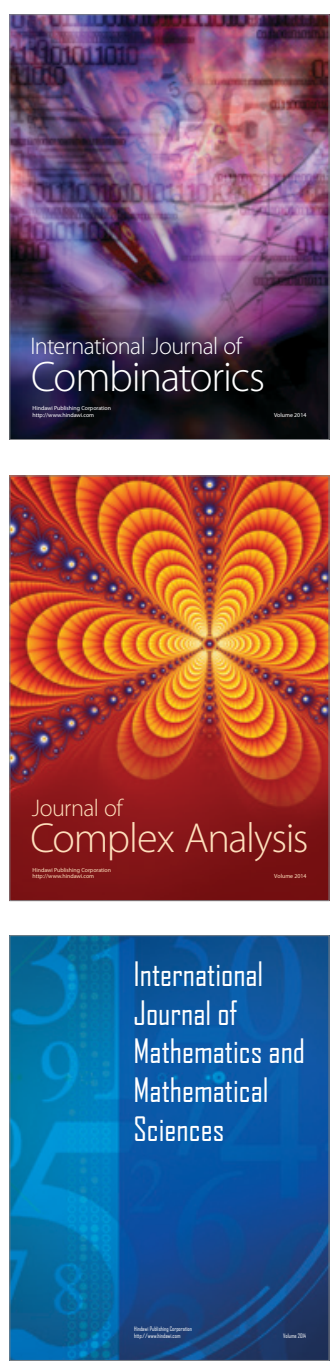
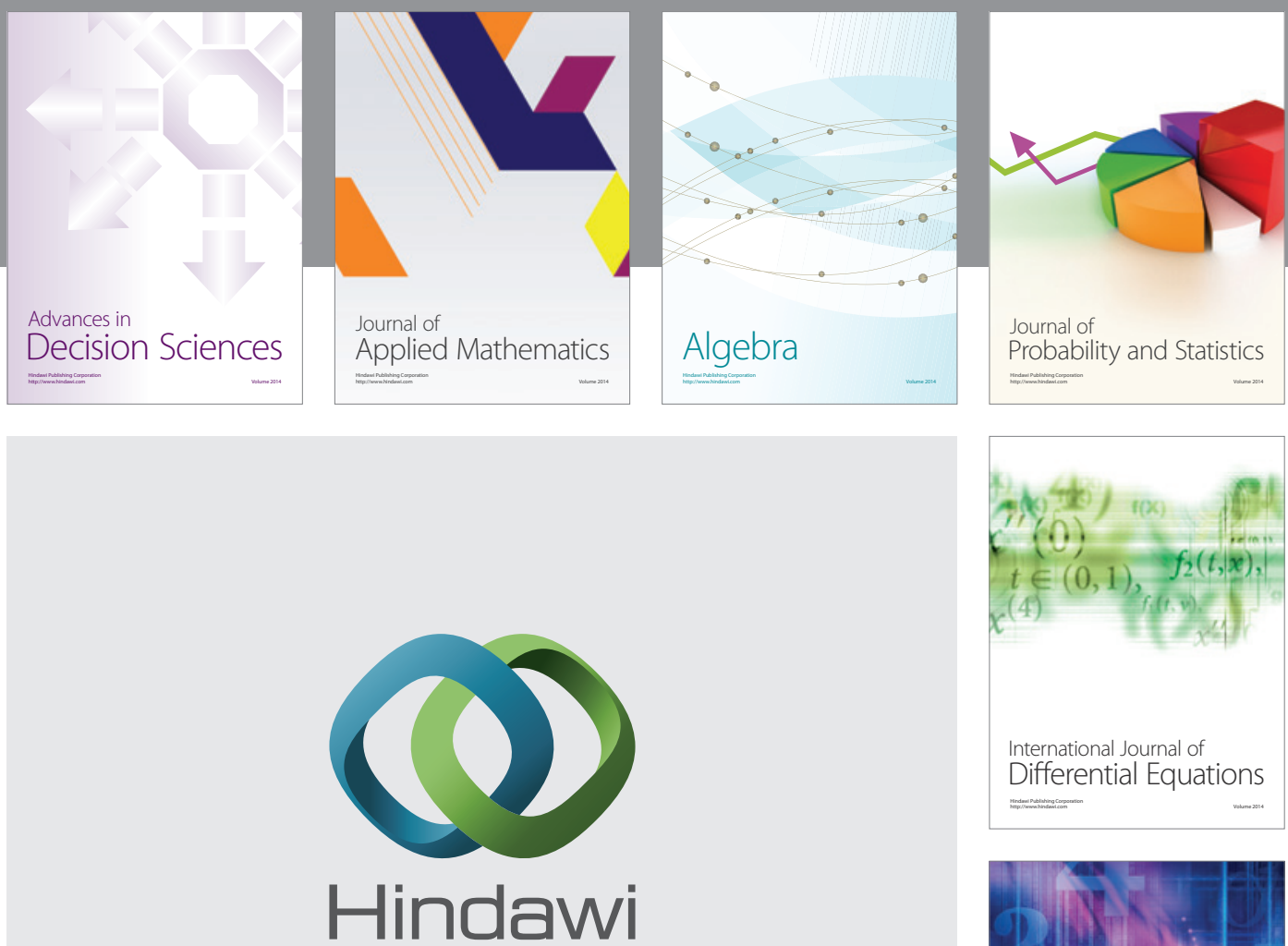

Submit your manuscripts at http://www.hindawi.com
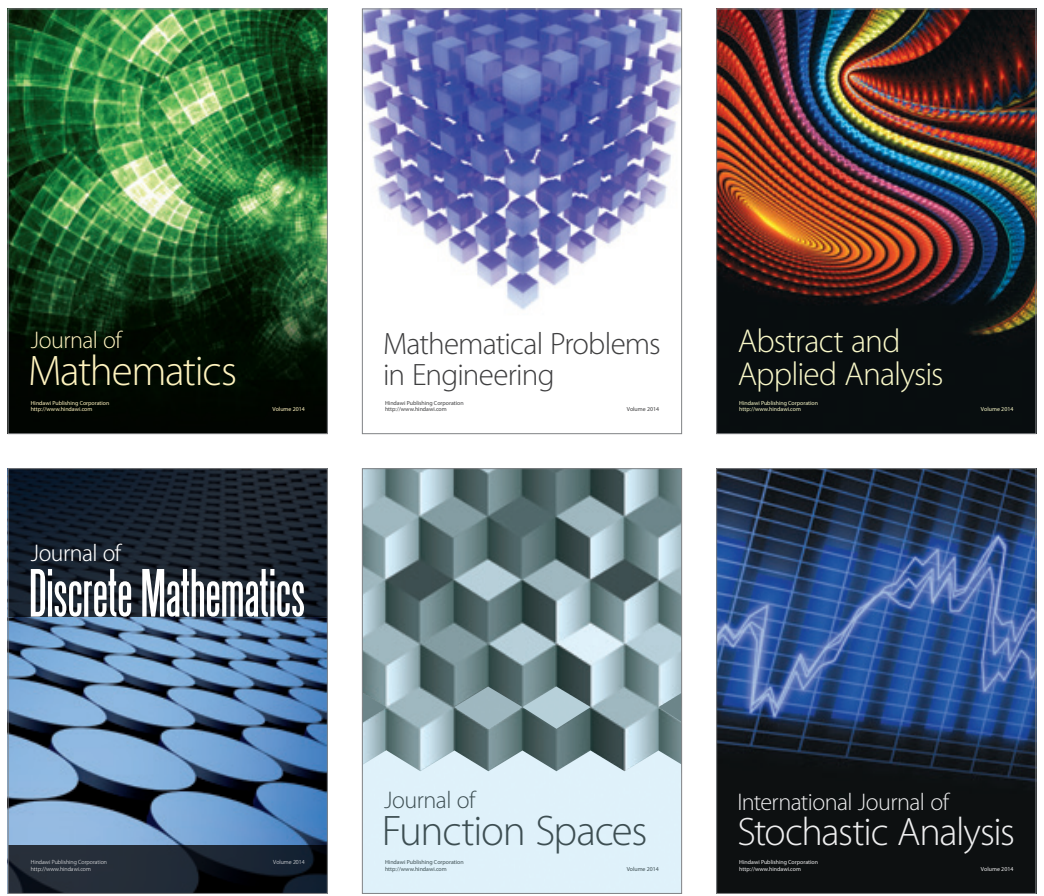

Journal of

Function Spaces

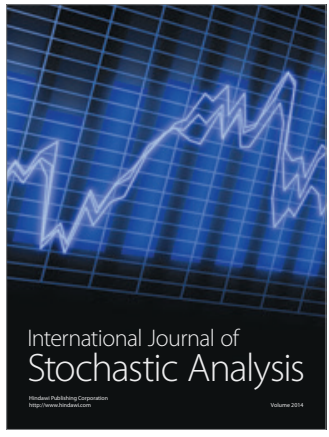

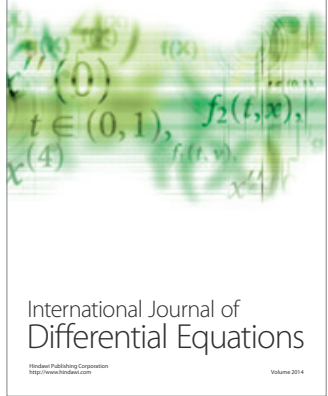
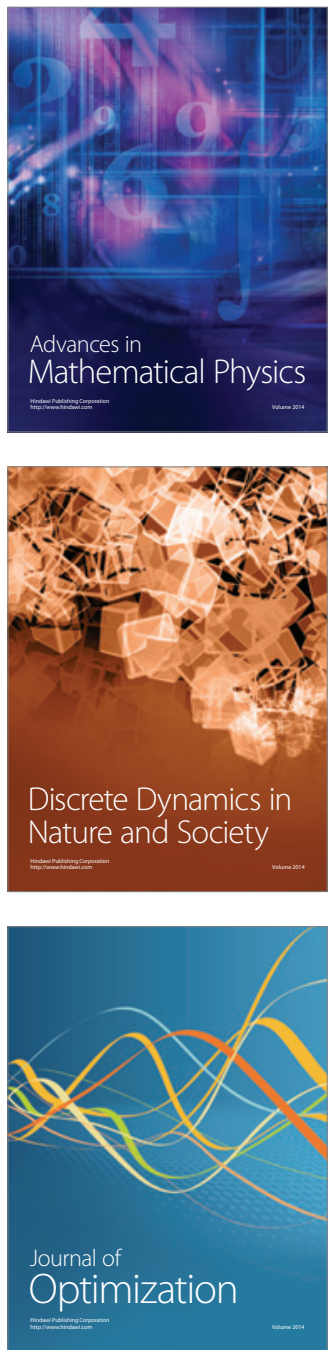